\title{
Statistical characterization of roughness uncertainty and impact on wind resource estimation
}

\author{
Mark Kelly and Hans E. Jørgensen \\ Wind Energy Division/Meteorology Section, Risø Lab./Campus, Danish Technical University, \\ Roskilde 4000, Denmark \\ Correspondence to: Mark Kelly (mkel@dtu.dk)
}

Received: 10 October 2016 - Discussion started: 1 November 2016

Revised: 20 January 2017 - Accepted: 21 February 2017 - Published: 25 April 2017

\begin{abstract}
In this work we relate uncertainty in background roughness length $\left(z_{0}\right)$ to uncertainty in wind speeds, where the latter are predicted at a wind farm location based on wind statistics observed at a different site. Sensitivity of predicted winds to roughness is derived analytically for the industry-standard European Wind Atlas method, which is based on the geostrophic drag law. We statistically consider roughness and its corresponding uncertainty, in terms of both $z_{0}$ derived from measured wind speeds as well as that chosen in practice by wind engineers. We show the combined effect of roughness uncertainty arising from differing wind-observation and turbine-prediction sites; this is done for the case of roughness bias as well as for the general case. For estimation of uncertainty in annual energy production (AEP), we also develop a generalized analytical turbine power curve, from which we derive a relation between mean wind speed and AEP. Following our developments, we provide guidance on approximate roughness uncertainty magnitudes to be expected in industry practice, and we also find that sites with larger background roughness incur relatively larger uncertainties.
\end{abstract}

\section{Introduction}

Microscale flow models have been employed for decades in wind energy assessment to estimate resources at one location based on wind measurements at a different site (Troen and Petersen, 1989). Furthermore, it has become increasingly popular in the past decade to use mesoscale model output to drive microscale models for the same purpose (e.g., Badger et al., 2014). Such flow modeling relies on characterization of the surface, including terrain elevation and surface roughness. As input to atmospheric flow models, both terrain elevation and roughness have uncertainties associated with their assignment. In practice, terrain elevation uncertainty tends to be dominated by the resolution of elevation maps (e.g., Sørensen et al., 2012) ${ }^{1}$. In contrast, there are a number

\footnotetext{
${ }^{1}$ Currently (2016), microscale models typically have computational resolutions finer than elevation maps; commonly available elevation maps in most of the world today have typical resolutions of $\Delta x \sim 10-90 \mathrm{~m}$, whereas quasi-linear (e.g., WAsP) and Reynoldsaveraged Navier-Stokes (RANS) models employed for wind are
}

of significant uncertainties associated with roughness, which do not (necessarily) depend on resolution; these include determination of roughness length $z_{0}$ from measurements and assignment of $z_{0}$ in industrial practice (based on land use, terrain type, and/or experience, for example). Overall, uncertainty related to roughness tends to be dominant over elevation-related uncertainty, particularly in wind-energy applications. In this work we develop a practical treatment of the effect of roughness uncertainty upon wind resource estimation, providing a formulation for estimation of roughnessinduced uncertainty in annual energy production.

First we review the definition of roughness length, introducing and demonstrating the statistical character of $z_{0}$, i.e., distributions of $z_{0}$ from measurements and the behavior of such; we statistically connect this to a practical un-

most often run with resolutions (much) finer than $10 \mathrm{~m}$. There are a growing number of exceptions, stemming from the advent of airborne laser-based terrain measurements that can offer resolutions less than $1 \mathrm{~m}$ (e.g., Zhang et al., 2005; Danish Geodata Agency, 2015). 
certainty metric. Then we present the theoretical framework that is used for wind resource estimation based on the geostrophic drag law (as used in the European Wind Atlas (EWA) methodology; Troen and Petersen, 1989) and including its relation to roughness. In Sect. 3 we introduce uncertainty; this includes basic characterization of the uncertainties inherent in (1) the roughness definition and observed distributions of $z_{0}$ (Sect. 3.1.1) and (2) the variations in $z_{0}$ prescribed in the wind energy industry (Sect. 3.1.2). We continue by showing how uncertainty in the background roughness can be translated into uncertainty in predicted wind distributions, within the European Wind Atlas framework (Sect. 3.2.1); here we provide derivations of the sensitivity of predicted winds to input roughnesses at observation and prediction sites. Consequently, we examine the effect of userassigned biases in roughness assignment and more generally the combined effect of (independent) roughness uncertainties on predicted wind speeds. For practical use we also develop an analytical relation between rated power, mean wind speed (Weibull- $A$ parameter), and AEP; this is accomplished via convolution of a generalized analytical power-curve form and Weibull wind distribution. Thus, we translate $z_{0}$ uncertainty into uncertainty of annual energy production (AEP).

Though there are different methods possible for determining or calculating roughness length, we concentrate here on the propagation of uncertainty in background roughness to predicted wind speeds and annual energy production. More details about and issues arising from alternate methods of roughness length calculation are beyond the scope of this article and are the basis of concurrent work to be included in a separate paper(s).

Lastly, we discuss approximate roughness uncertainty magnitudes expected in practice and the consequences of them. This also includes, for example, the result that sites with larger background roughness tend to give larger relative uncertainty (i.e., \%) in predicted wind speeds and significant uncertainty in AEP. We also discuss implications for the use of mesoscale simulation data for driving microscale models, i.e., generalization of wind statistics.

\section{Basis and framework}

Physically, this work simply considers the use of wind measurements (statistics) at some height above ground level at one location in order to predict wind statistics at another location and height. Starting with ideal (uniform flat) terrain, this prediction can be broken into components, commonly labeled within the wind resource assessment community as vertical and horizontal extrapolation. Subsequently, the theoretical foundation of this work involves the two basic components related to the physics modeled by such extrapolations: these are the wind profile for vertical extrapolation and the geostrophic drag law (GDL) for relating the wind statistics at different sites; they are covered in Sect. 2.1 and 2.2, respectively. The vertical wind profile form (of which the simplest is the logarithmic law) requires a surface roughness length, and the GDL also requires a characteristic (background) roughness length. Because we wish to relate uncertainty in roughness to uncertainty in wind energy estimates, i.e., finding the uncertainty in accounting for the effect of the surface, we first begin by examining roughness length, both in theory (i.e., definition) and in practice (e.g., its statistical character).

\subsection{Roughness length: theory and practice}

The concept of roughness length began with characterization of the velocity profile in ideal engineering flows (e.g., pipes), where roughness has a direct physical interpretation (Nikuradse, 1933; Tripp, 1936); it was further adopted to describe the wind profile in the atmospheric surface layer (ASL), whereby it has an implicit (and not directly physical) definition (Monin and Yaglom, 1971). The basic role of roughness length and its definition, can be seen through the ideal expression for the mean wind profile $U(z)$ over a homogeneous flat surface in neutral conditions (without thermal stability effects):

$U(z)=\frac{u_{*}}{\kappa} \ln \left(\frac{z}{z_{0}}\right)$.

In Eq. (1) $z_{0}$ is the roughness length and $z$ the height above (distance normal to) the surface, expressed in the same units; $\kappa$ is the von Kármán constant, generally accepted to be 0.4 (Högstrom, 1996). The friction velocity $u_{*}$ is defined by $u_{*}^{2} \equiv-\left\langle u^{\prime} w^{\prime}\right\rangle$, i.e., as mean momentum transport towards the surface through turbulent stream-wise $\left(u^{\prime}\right)$ and vertical or surface-normal $\left(w^{\prime}\right)$ velocity fluctuations. The roughness $z_{0}$ can also be seen as an integration constant since Eq. (1) results from integrating $\mathrm{d} U / \mathrm{d} z=u_{*} /(\kappa z)$; the latter is typically derived via dimensional analysis, through the Buckingham Pi theorem (e.g., Stull, 1988; Wyngaard, 2010). The logarithmic wind profile (Eq. 1) depends upon a number of assumptions: $u_{*}$ is effectively constant from the surface up to height $z$ (i.e., $\mathrm{d} u_{*}^{2} / \mathrm{d} z \ll \sigma_{u}^{2} / \ell_{\mathrm{ABL}}$ Wyngaard, 2010), the surface is flat and uniform, there is horizontal homogeneity (no variations parallel to the surface), there is no height dependence in the forcing of the flow, and there are (no effects due to) temperature variations, i.e., the only variables determining $\mathrm{d} U / \mathrm{d} z$ are $u_{*}$ and $z$.

\subsubsection{Calculation of roughness length from wind measurements}

From Eq. (1) one can see that for $U$ measured at two heights $\left\{z_{1}, z_{2}\right\}$, the roughness can be calculated by

$\ln \left(z_{0}\right)=\frac{U\left(z_{2}\right) \ln z_{1}-U\left(z_{1}\right) \ln z_{2}}{U\left(z_{2}\right)-U\left(z_{1}\right)}$.

While one can also obtain the roughness via the shear exponent (e.g., Kelly et al., 2014a) that is often used in wind 
energy, Eq. (2) does not involve approximations and directly follows from the definition of roughness. One can also use friction velocity measured in the surface layer and wind speed from one (or more) height(s) to derive roughness (e.g., $z_{0}=z \exp \left[-\kappa U(z) / u_{*}\right]$ from Eq. 1), but doing so requires sonic anemometers, which are not yet commonly used in the wind energy industry. Thus, we use Eq. (2) for the observed roughness data analyzed and shown in this paper, and leave alternate $z_{0}$ estimation methods for concurrent work and dissemination that focuses solely upon roughness. This choice is further supported by the focus of the present article - we are concerned here with the impact of roughness length on wind energy estimates - and because we develop and use an uncertainty-estimation framework that is generally applicable to $z_{0}$, regardless of whether $z_{0}$ is derived from Eq. (2) or via $U / u_{*}$.

\subsubsection{Roughness as a statistic}

Even in seemingly ideal conditions - such as measuring wind profiles in the surface layer at a site where the terrain is flat and appears uniform, with non-neutral cases excluded - in practice one still observes a broad range of roughnesses. This is demonstrated in Fig. 1, which shows the roughness length calculated from 10 and $40 \mathrm{~m}$ measurements at the Danish National Wind Turbine Test Station at Høvsøre for upwind directions corresponding to flat and homogeneous surfaces (east of the meteorological measurement mast). Here we have filtered out non-neutral conditions by keeping only cases unaffected by thermodynamic stability by using $z /|L|<0.001$, i.e., for Obukhov lengths $L$ much greater than the heights of measurement.

Figure 1 starkly demonstrates that even at a homogeneous, well-studied, and presumably simple site, roughness length has a distribution of significant width. Note that we plot the distribution of roughness length in logarithmic space; this is done because it is $\ln \left(z_{0}\right)$ that directly affects the wind profile, as in Eq. (1). This also highlights the breadth of the distribution (several orders of magnitude) and that we must subsequently approach roughness uncertainty in a $\mathrm{mul}$ tiplicative (dimensionless) way and not in an additive way. We also remind that the roughness lengths generally used in wind flow modeling and resource assessment actually correspond to some geometric mean, which should be based on the $z_{0}$ distribution (alternately one can express wind profiles in terms of the distribution $P\left(\ln z_{0}\right)$ and corresponding arithmetic mean; cf., Kelly and Gryning, 2010); unfortunately $z_{0}$ is not (yet) defined explicitly as such in typical wind engineering practice. Thus, in this paper we focus on roughness uncertainty within the "implied mean-roughness" framework implicit in standard wind engineering.

In addition to the relatively wide distribution apparent for roughnesses obtained from $30 \mathrm{~min}$ averages shown in Fig. 1 (and slightly wider for $10 \mathrm{~min}$ averages, not shown), one can also see some local - and nonideal - details. One sees the minor effects of a barn and a small building located roughly $800 \mathrm{~m}$ upwind at $\sim 80$ and $\sim 110^{\circ}$, respectively, as well as the larger effect of the seasonally varying marsh-fjord coastline $800-900 \mathrm{~m}$ to the southeast $\left(\sim 130-135^{\circ}\right)$. Such roughness changes tend to violate the assumptions behind the logarithmic profile over a range of observation heights falling within the nonequilibrium internal boundary layer (IBL) transition region (Sempreviva et al., 1990; Bou-Zeid et al., 2004; Calaf et al., 2014) ${ }^{2}$. The more drastic semi-coastal roughness change contaminates the shear measured between 10 and $40 \mathrm{~m}$ enough to give the larger apparent $z_{0}$ shown in Fig. $1 \mathrm{~b}$ as $\phi \rightarrow 135^{\circ}$ and the subsequently wider distribution $P\left(z_{0}\right)$ shown in Fig. 1a for the $120^{\circ}$ sector.

Because neutral conditions tend to be encountered most often (stability distributions have their peak around $L^{-1}=0$; see Kelly and Gryning, 2010), the distribution of shear exponent $P(\alpha)$ can also be related in terms of an effective roughness length without filtering stability to exclude non-neutral conditions (Kelly et al., 2014a). Thus, the wind profile can indeed give information about the surface, though the shear at higher $z$ includes the effect of increasingly more terrain further upwind (potentially including hills as well as roughnesses) ${ }^{3}$.

Avoiding substantial changes in surface characteristics and/or land use, this can be useful towards the aim of gauging background $z_{0}$.

One can also calculate a more local roughness length via Eq. (1) using measurements of $U$ and $u_{*}$ within the surface layer (filtering out non-neutral conditions via measured heat fluxes), but doing so requires sonic anemometers, which are not (yet) commonly used in the wind industry. For example, using $U$ and $u_{*}$ measured at $z=10 \mathrm{~m}$ for the case above gives $P\left(z_{0}, \phi\right)$ that is insensitive to the inhomogeneities described above, i.e., it does not jump as $\phi$ increases above $\sim 130^{\circ}$. Although the resultant $z_{0}\left(U / u_{*}\right)$ tends to better conform to the assumptions behind surface-layer theory and Eq. (1), it is consequently limited to ASL heights - which in stable conditions (e.g., nighttime, winter) only extend to $\sim 10-20 \mathrm{~m}$. Furthermore, the $z_{0}$ derived from $U / u_{*}$ in the ASL is local, only pertaining to the nearest several hundred meters, perhaps less in stable conditions. However, the widths of $P\left(\ln z_{0}\right)$ derived from $U / u_{*}$ (not shown) are on par with those obtained from $U$ at two heights and displayed in Fig. 1.

Thus, in the present article concerned about uncertainty, we do not address the implications of surface-layer theory

\footnotetext{
${ }^{2}$ The IBL develops downwind from a roughness change with expansion slope $(z: x)$ of roughly $1: 100$, and the top of the associated transition region expands at a variable rate of 1 to $\sim 12-15$. For the example noted here, this corresponds to the flow measured by anemometers at both 10 and $40 \mathrm{~m}$ being affected.

${ }^{3}$ The increasing area of surface affecting winds at increasing heights, and also associated averaging issues, is beyond the scope of the current article (consult, e.g., Lettau, 1969; Garratt, 1978; Hasager and Jensen, 1999).
} 

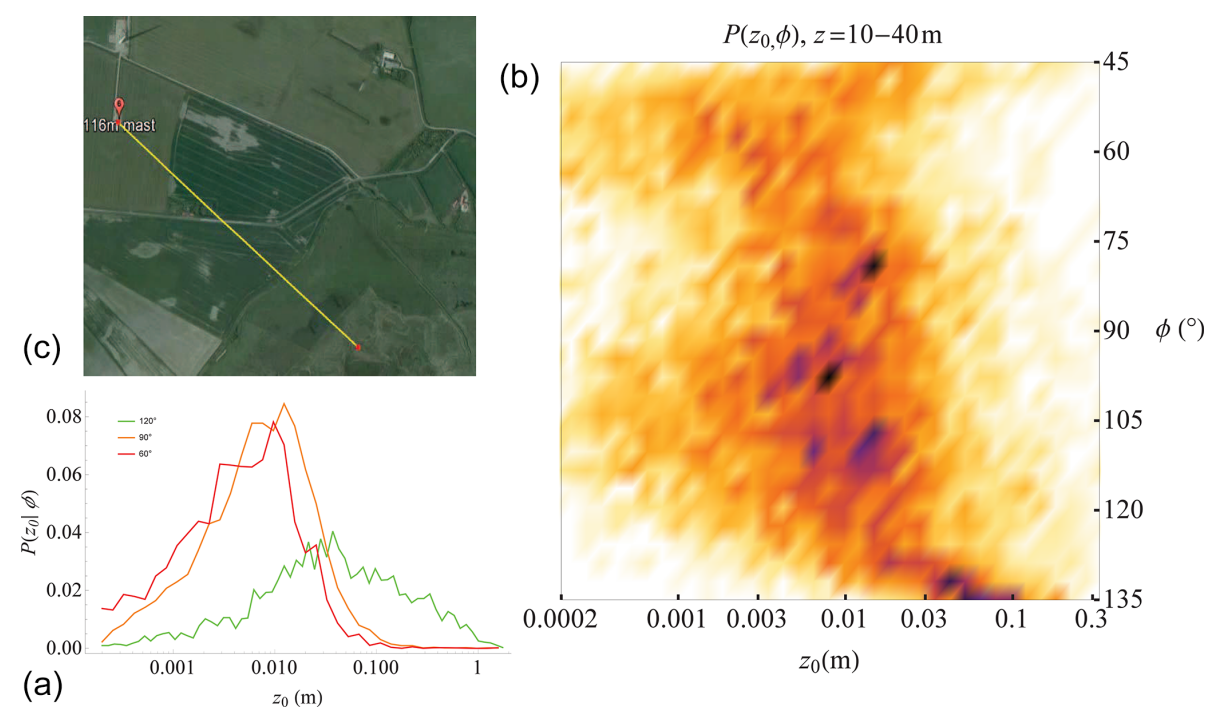

Figure 1. (a) Distribution of $z_{0}$ for homogeneous land sectors $\left(30^{\circ}\right.$ wide) east of Høvsøre. (b) Joint distribution of $z_{0}$ and wind direction $\phi$; darker represents most common values, and white is no occurrence. Calculation follows Eq. (2), with $z_{1}=10$ and $z_{2}=40 \mathrm{~m}$, and it is limited to neutral conditions $\left(|L|^{-1}<0.001 \mathrm{~m}^{-1}\right.$ ). (c) Visual map east of site (red pointer; southern border of homogeneous zone at $\sim 130^{\circ}$ denoted by yellow line).

nor its conditional violation, but rather focus on the effect of roughness uncertainty - as it would be measured (or assigned) in industrial practice - upon resource assessment, particularly through horizontal extrapolation from an observation mast to a separate turbine location(s).

\subsection{Geostrophic drag law: European Wind Atlas method}

The geostrophic drag law (GDL) allows wind statistics observed at one site to be applied at potential wind farm sites nearby that may have different surface characteristics (i.e., roughness and terrain elevation); it is the basis of the EWA method (Troen and Petersen, 1989) used widely for wind resource estimation. The GDL arises from matching the dimensionless surface-layer profile of mean wind in neutral conditions (i.e., the log law divided by $u_{*}$ ) to dimensionless solutions of the mean horizontal equations of motion away from the surface, as affected by the Coriolis force (Ellison, 1956; Krishna, 1980; Walmsley, 1992). The mean atmospheric boundary layer (ABL) flow is driven by a large-scale mean pressure gradient $\nabla P$, also expressible as the geostrophic wind $\mathbf{G} \equiv-\hat{\boldsymbol{k}} \times \nabla P /(f \rho)=$ $\{-\partial P / \partial y, \partial P / \partial x\} /(f \rho)$, where $\hat{\boldsymbol{k}}$ is the vertical unit vector and $f$ is the latitude-dependent Coriolis parameter; the pressure gradient force is balanced (vectorially) by the Coriolis force and momentum transfer to the surface. Thus, the GDL essentially relates the large-scale forcing (expressible as the geostrophic wind above the ABL) to the surface-layer momentum flux (friction velocity), depending on the surface roughness.
The geostrophic drag law can be simply expressed in scalar form as

$G=\frac{u_{*}}{\kappa} \sqrt{\left[\ln \left(\frac{u_{*} / f}{z_{0}}\right)-A_{0}\right]^{2}+B_{0}^{2}}$,

where $A_{0}$ and $B_{0}$ are empirical constants (taken by the EWA to be 1.8 and 4.5 ). Thus, for two sites that can be assumed to have the same large-scale forcing (distribution of $\mathbf{G}$ ), then the wind statistics at one site can be translated to wind statistics at the other. From the wind profile relation (Eq. 1) one can obtain $u_{*}$ from measured $U$ over one roughness $z_{0,1}$, and subsequently $G$ from Eq. (3); then at the prediction site one can solve Eq. (3) to get $u_{*}$ at a potential turbine site and subsequently find $U$ there over a roughness $z_{0,2}$. Below, we will show the impact of roughness uncertainty upon wind speed and AEP estimates via Eqs. (1) and (3).

\section{Uncertainty}

\subsection{Roughness and uncertainty components}

In general, uncertainty can be classified into two types (Kiureghian and Ditlevsen, 2009): aleatoric uncertainty, and epistemic uncertainty.

First, aleatoric (sometimes called statistical or random) uncertainty is the variability in a quantity that arises from randomness inherent in the process(es) that impact said quantity. Epistemic or systematic uncertainty arises due to lack of knowledge about a quantity (imperfect understanding of it in the real world).

The aleatoric (random) uncertainty inherent in roughness length can be said to include that associated with the width 
of the observed distribution of $z_{0}$ shown in Sect. 2.1.2. This tends to be due to variability in the system being described; the system in this case is the atmospheric surface layer and the surface nearby the measurement point that influences the flow. However, there is also an epistemic component contained within the distributions $P\left(z_{0}\right)$ shown in Fig. 1; it is due to effects that were neglected in the derivation of the theory used, namely the logarithmic law (Eq. 1). Physically, this includes inhomogeneities in the surface upwind and dependence of surface characteristics upon wind speed (i.e., water or flexible vegetation; see Monin and Yaglom, 1971); within the context of the turbulent surface layer as described by turbulence theory, it tends to be manifested via turbulent transport (Kelly et al., 2014a; Sogachev and Kelly, 2016).

When performing resource assessment, in practice wind engineers characterize the surface via roughness length (as well as terrain elevation, which we do not treat in this paper). Roughness characterization can occur via assignment of $z_{0}$ values chosen by the wind engineer or through roughness values (or land-use types) inherited from maps acquired from a third party. Typically, the former has dominated the wind industry, though the latter is becoming more common; land-use types and classes are contained in some geographical data products, but these have not yet been shown to be consistently or universally translatable to roughness lengths for different parts of the world (see, e.g., Marticorena et al., 2006; Torbick et al., 2006). Either way, epistemic uncertainty arises due to our ignorance of the appropriate representative roughness length ${ }^{4}$ and is introduced when characterizing the surface via a single roughness; this uncertainty exists regardless of whether the characteristic $z_{0}$ is chosen by an algorithm assigning values to a map based on look-up tables for various land classifications or by a wind engineer who has visited the (potential) site.

The epistemic components associated with the theory used to convert; wind observations into observed $z_{0}$ tend to manifest via turbulent transport and subsequently behave randomly, arising to a good degree of variability of the surface itself (hence being debatably aleatoric). These are in contrast to the uncertainty arising from selection of $z_{0}$ by engineers or the uncertainty inherent in (usage of) a relatively small number of widely used sources for roughness maps, which can contain significant bias and are not (directly) related to measurement. Thus, here we group the former, observationally related uncertainty together with the aleatoric uncertainty, and then separately consider the epistemic uncertainty implicit in assignment of roughness values by wind engineers in practice.

\footnotetext{
${ }^{4}$ As shown in the section above, the representative roughness length should be based on a geometric mean, due the $\ln \left(z_{0}\right)$ behavior exhibited by the surface-layer wind profile in neutral conditions.
}

\subsubsection{Uncertainty in observation-based $z_{0}$}

For the observation-based roughness lengths displayed in Sect. 2.1.2 (Fig. 1), the distributions are best described (and thus plotted) as $P\left(\ln z_{0}\right)$, again consistent with both the $\ln \left(z_{0}\right)$ behavior expected within the wind profile and with the geometric (multiplicative) averaging needed to obtain a characteristic mean roughness. The width of the $\ln \left(z_{0}\right)$ distributions shown in Fig. 1 gives indication of the variability in $\ln z_{0}$ over many $30 \mathrm{~min}($ or $10 \mathrm{~min})$ periods. In particular the $P\left(\ln z_{0}\right)$ for $30^{\circ}$-wide directional sectors can be considered, that is $P\left(\ln z_{0} \mid \varphi\right)$, since sectors of this width are commonly used in resource assessment. The homogeneous 60 and $90^{\circ} \mathrm{sec}-$ tors at Høvsøre (Fig. 1) have similar shapes, and both exhibit half-peak widths of roughly one-half order of magnitude (a factor of $\sim 3$ ); i.e., for a given sector's background roughness $z_{0}$, the width of the distribution can be seen as that defined roughly between $z_{0} / 3$ and $3 z_{0}$.

However, the uncertainty in determining a representative roughness length - via the appropriate (geometric) mean - is not the same as the width of the $\ln \left(z_{0}\right)$ distribution. Rather, the uncertainty in the mean roughness is the width of the distribution of expected means calculated for a given site and sector. For this purpose we use a basic "bootstrap" resampling method (Varian, 2005; Wu, 1986): simply resampling randomly from the diagnosed (30 $\mathrm{min}$ ) roughness lengths, we synthesize a distribution of $10^{5}$ values of geometric-mean roughness $\left(\left\langle z_{0}\right\rangle_{g}\right)$ per sector. This results in a log-normal distribution of mean $z_{0}$ (Gaussian distribution of $\left.\ln z_{0}\right)$; this distribution $P\left(\exp \left[\left\langle\ln z_{0}\right\rangle\right]\right)$ is centered around a value equal to the geometric mean that had been found for each sector by operating directly on the wind data. The width of each (sector-wise) distribution of mean roughnesses from resampling depends on the number of resampled points used to create each mean in the synthesized distribution. For a number equivalent to 1 year's worth of data (based on the sector-wise frequency of occurrence), the mean distributions are in fact much narrower than the distributions shown in Fig. 1. The bootstrapped mean-roughness distribution is almost perfectly fit by a log-normal form; the half width $w_{\left\langle z_{0}\right\rangle_{\mathrm{RS}}}$ for this form can be simply expressed non-dimensionally (i.e., effectively normalized by the expected mean) via the standard deviation of mean $\ln z_{0}$ from resampling $\left(\sigma_{\left\langle\ln z_{0}\right\rangle_{\mathrm{RS}}}\right)$ as

$$
\frac{w_{\left\langle z_{0}\right\rangle_{\mathrm{RS}}}}{\left\langle\left\langle z_{0}\right\rangle_{\mathrm{RS}}\right\rangle_{g}}=\exp \left\{\sigma_{\left\langle\ln \left[z_{0} /\left\langle z_{0}\right\rangle_{g}\right]\right\rangle_{\mathrm{RS}}}\right\}=\exp \left\{\sigma_{\left\langle\ln z_{0}\right\rangle_{\mathrm{RS}}}\right\}-1
$$

For the Høvsøre homogeneous land sectors treated here and the bootstrapped means, each calculated from 1 year's worth of resampled data, the $w_{\left\langle z_{0}\right\rangle_{\mathrm{RS}}}$ of the sector-wise distributions of these means are about $5 \%$ of the expected mean roughness length (specifically, 5.4, 4.1, and $5.3 \%$ of the respective $\left\langle\left\langle z_{0}\right\rangle_{\mathrm{RS}}\right\rangle$ in each sector from Eq. 4). Thus, considering only calculations of $z_{0}$ from wind speeds measured at two (10 and $40 \mathrm{~m}$ ) heights via Eq. (2) from 1 year of data, the roughness uncertainty for the three sectors shown in Fig. 1 
Table 1. Means (geometric and arithmetic) and corresponding deviations in $z_{0}$, surveyed from two groups of wind resource experts for the two terrain types shown in Fig. 2. For conventional (linear) standard deviation $\sigma_{z_{0}}$, number in parenthesis is $\sigma_{z_{0}} /\left\langle z_{0}\right\rangle$, given for comparison with the logarithmic standard deviation $\left(\exp \left\{\sigma_{\ln \left[z_{0} /\left\langle z_{0}\right\rangle_{g}\right]}\right\}\right)$.

\begin{tabular}{lcc|cc|cc|rrr}
\hline & \multicolumn{2}{c}{ Geom. mean, $\left\langle z_{0}\right\rangle_{g}$} & \multicolumn{2}{|c|}{ Arith. mean, $\left\langle z_{0}\right\rangle$} & $\exp \left\{\sigma_{\ln \left[z_{0} /\left\langle z_{0}\right\rangle_{g}\right]}\right\}$ & \multicolumn{3}{|c}{ Standard Deviation, $\sigma_{z_{0}}$} & \\
\cline { 2 - 7 } Group & Grass & Forest & Grass & Forest & Grass & Forest & Grass & Forest & $n$ \\
\hline Vindkraft-net & $4.0 \mathrm{~cm}$ & $0.87 \mathrm{~m}$ & $5.6 \mathrm{~cm}$ & $1.6 \mathrm{~m}$ & $124 \%$ & $162 \%$ & $6.4 \mathrm{~cm}(115 \%)$ & $2.5 \mathrm{~m}(158 \%)$ & 28 \\
DTU Wind & $4.2 \mathrm{~cm}$ & $0.82 \mathrm{~m}$ & $5.5 \mathrm{~cm}$ & $1.0 \mathrm{~m}$ & $112 \%$ & $113 \%$ & $4.7 \mathrm{~cm}(86 \%)$ & $0.57 \mathrm{~m}(57 \%)$ & 19 \\
Combined & $4.1 \mathrm{~cm}$ & $0.85 \mathrm{~m}$ & $5.6 \mathrm{~cm}$ & $1.3 \mathrm{~m}$ & $117 \%$ & $141 \%$ & $5.7 \mathrm{~cm}(103 \%)$ & $2.0 \mathrm{~m}(146 \%)$ & 47 \\
\hline
\end{tabular}

is about $5 \%$. For longer data sets, the uncertainty decreases; for example, randomly drawing from the entire 10-year set leads to half widths of $1-2 \%$.

One should be reminded that there are other methods to calculate $z_{0}$, such as using the surface-layer friction velocity $u_{*}$ and wind speed at one (or more) measurement height(s) via Eq. (1), which may result in different values of estimated mean and/or characteristic roughness length. For example, repeating the analysis above using Eq. (1) with $U$ and $u_{*}$ measured at $10 \mathrm{~m}$ height, we again obtain well-behaved distributions of bootstrapped mean roughness whose half widths are about $5 \%$; one might take this as the implied uncertainty. However, the mean values (for a given sector) can actually differ between the two methods by an amount that can greatly exceed 5\% (in these Høvsøre land sectors they can differ by a factor of $\sim 3$ !). This difference is related to the flow physics at increasing distances from the mast (the momentum flux footprint), the details of which are beyond the scope of this paper; we defer further discussion of such differences to Sect. 4.

\subsubsection{Uncertainty and ensembles of user input}

Even for an ideal homogeneous landscape, the wind industry, which is a collection of wind engineers and companies, will as a group assign different roughnesses to characterize the surface (whether actively or inherited via acquired maps). This results in a distribution of $z_{0}$ assigned to predict the wind for any given site, and in effect to an (epistemic) uncertainty, and subsequently industry-wide variation in predicted AEP, even at the most simple sites.

We provide a simple practical example of gauging such epistemic uncertainty based on a systematic exercise: we asked separate groups of wind resource assessment experts to individually evaluate the surface roughness length for two commonly encountered land surface types. The groups of participants in this exercise were polled at meetings of the Danish Wind Power Network "Vindkraft-Net" (Kelly and Jørgensen, 2014) and of the Meteorology section of the Department of Wind Energy (Risø lab/campus) in the Danish Technical University; their backgrounds and foci range from

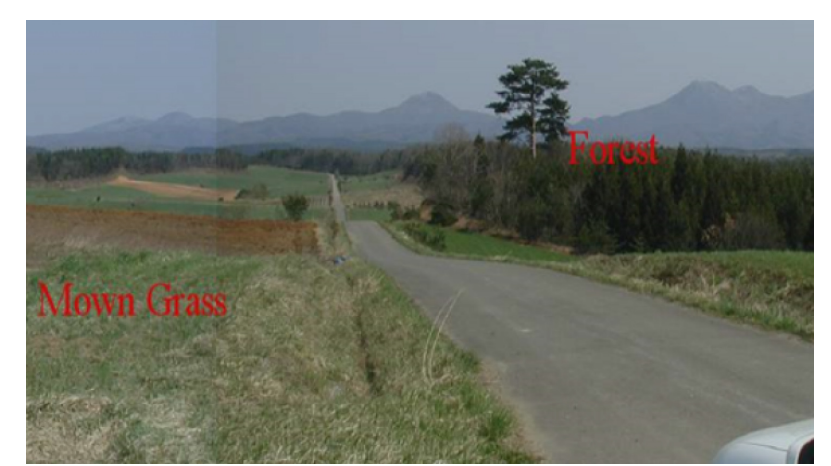

Figure 2. Image of the two areas (grassy and forested) used in roughness survey exercise.

wind engineering and commercial site assessment to research boundary-layer meteorology and wind resource calculation.

The participants were shown a picture containing both a grassy area and a forested area (the latter specified as having a mean tree height of $15 \mathrm{~m}$ ) and were asked to give $z_{0}$ for each of these two areas; the picture is replicated in Fig. 2. The raw results of the roughness survey, which consisted of 19 and 28 participants are shown in Table 1.

Note that Table 1 includes not only a geometrically defined mean $\left\langle z_{0}\right\rangle_{g} \equiv\left[\prod_{i}^{n}\left(z_{0}\right)_{i}\right]^{1 / n}=\exp \left[\sum_{i}^{n} \ln \left(z_{0}\right)_{i} / n\right]$ and associated dimensionless standard deviation $\exp \left\{\sigma_{\ln \left[z_{0} /\left\langle z_{0}\right\rangle_{g}\right]}\right\}$ that are consistent with the logarithmic definition of roughness, but also the commonly used arithmetic mean and (normalized) standard deviation of user-estimated $z_{0}$. The latter statistics are included for comparison and because (in contrast to the flow physics) there is some tendency for wind engineers to think linearly rather than logarithmically. As can be seen in Table 1, the arithmetic (linear) mean of $z_{0}$ is unsurprisingly larger than the properly (logarithmically) averaged $z_{0}$, by $\sim 30-40 \%$ for grass and $\sim 20-80 \%$ for forest. Arithmetic calculation of $z_{0}$ statistics subsequently tends to give a smaller normalized deviation compared to the proper log-rms statistic for the raw surveyed data, particularly as the $z_{0}$ distribution is dominated by values smaller than $1 \mathrm{~m}$ (expected from the mathematical character of geometric $\left[\ln z_{0}\right]$ vs. arithmetic averages). Overall, the variability in polled 
Table 2. Bootstrapped statistics of mean roughnesses from (resampled) user-provided $z_{0}$ given by two groups of wind resource experts, using three resampled values per mean calculation; data are for the two terrain types shown in Fig. 2.

\begin{tabular}{lcc|cc}
\hline & \multicolumn{2}{c}{ Geom. mean, $e^{\left\langle\ln \left\langle z_{0}\right\rangle_{g}, \mathrm{RS}\right\rangle_{g}}$} & \multicolumn{2}{|c}{ Eff. dev., $w_{\left\langle z_{0}\right\rangle_{\mathrm{RS}}}$} \\
\cline { 2 - 5 } Group & Grass & Forest & Grass & Forest \\
\hline Vindkraft-net & $4.0 \mathrm{~cm}$ & $0.87 \mathrm{~m}$ & $58 \%$ & $73 \%$ \\
DTU Wind & $4.2 \mathrm{~cm}$ & $0.82 \mathrm{~m}$ & $53 \%$ & $53 \%$ \\
Combined & $4.1 \mathrm{~cm}$ & $0.85 \mathrm{~m}$ & $56 \%$ & $65 \%$ \\
\hline
\end{tabular}

roughness lengths for the two cases is on the order of but larger than the expected roughness length itself, i.e., by a factor of $\sim 1.1-1.3$ times the estimated mean $z_{0}$ for grass or $\sim 1.1-1.6$ times the mean for the forest case. This might be taken as an estimate for uncertainty in $z_{0}$ for such cases.

The variability in the user data differs between the polled groups and might be affected by the limited sample size. Due to the limited distributions of polled roughness lengths (not shown) gathered from each of the two expert groups, an alternate estimate of collective user uncertainty (i.e., industrywide) is provided by again applying a resampling method to the distribution of surveyed $z_{0}$. Following the averaging of expert-elicited $z_{0}$ and the uncertainty characterization of the previous section, bootstrapping (Varian, 2005; Wu, 1986) is used to resample the elicited $z_{0}$ values and construct a distribution of the means. Calculating each mean from $n$ nonunique random data samples and repeating $10^{6}$ times, we generate distributions of $\overline{z_{0}}$ for the two cases. For $n \gtrsim 3$, we find log-normal distributions for the bootstrapped geometric mean $\left(\overline{z_{0}}=\left\langle z_{0}\right\rangle_{g}\right)$, as expected from the central limit theorem $\left(\ln \overline{z_{0}}\right.$ becomes Gaussian). In the limit of the sample data set being perfectly representative of wind industry practices, the bootstrapped distribution for a given $n$ is equivalent to the $P\left(\overline{z_{0}}\right)$ expected when any given wind engineer uses $n$ values to calculate the mean roughness for a site such as the grass or forest case used here. The means of the resampled distributions are the same as for the raw roughness samples in Table 1, regardless of $n$. The deviation, however, decreases with $n$. For $n=1$, the deviations converge to those in Table 1 , while the values of the effective geometric deviation $w_{\left\langle z_{0}\right\rangle_{\mathrm{RS}}}$ behave as approximately $1+n^{-0.53}$ (the deviations fall slightly more rapidly than $n^{-1 / 2}$ due to the slightly irregular sample or survey). As an example, Table 2 shows the geometric means and deviations for these mean distributions using $n=3$ for the two groups and cases considered.

From Table 2, one infers the seemingly obvious result that for users taking an average of three industry-accepted roughness estimates (assuming that they span the sample taken) instead of just one - the expected (industry-wide) uncertainty is reduced; we point out that such a conclusion depends on having reasonably representative roughness values to choose from.
To summarize, in this subsection we saw that the equivalent (normalized logarithmic) standard deviation from surveys of engineer or user-assigned roughness is of the order of the expected roughness itself, as shown in Table 1. In terms of Eq. (4), we expect an uncertainty equal to the half width of the (expected user input) distribution of $\ln z_{0}$ to be approximately $w_{\left\langle z_{0}\right\rangle_{g}} \sim\left\langle z_{0}\right\rangle_{g}$. In the following section we would like to show, in general, how uncertainty in $z_{0}$ - whether due to user input or measurement - propagates into wind speed and AEP estimates.

\subsection{Propagation of roughness uncertainty}

The uncertainty in roughness length has an effect on a number of key variables needed for wind resource assessment. Since the geostrophic wind depends upon the surface friction velocity $u_{*}$, in practice one must use a wind profile form (model) to translate measured wind statistics (e.g., Weibull$A$ or mean wind speed) into the corresponding $u_{*}$ analogue. This is typically accomplished by using the log law (Eq. 1), which is valid in statistically neutral conditions, and approximately in the mean (Kelly and Gryning, 2010; Kelly and Troen, 2016). Furthermore, to relate $u_{*}$ at the prediction site to the (mean) geostrophic wind $G$, Eq. (3) must somehow be solved for $u_{*}$. A direct analytical solution for $u_{*}(G)$ via Eq. (3) is not possible; thus, Jensen et al. (1984) developed the approximate "reverse geostrophic drag-law" form

$u_{*}(G) \simeq \frac{0.485 G}{\ln \left(G / f z_{0}\right)-A_{0}}$.

We adopt Eq. (5) and use it along with Eqs. (1) and (3) in order to relate wind speeds and roughness lengths for a given pair of prediction and measurement sites.

\subsubsection{Sensitivity of predicted wind speed to background roughnesses}

By using the logarithmic wind profile (Eq. 1) at both measurement and prediction locations, along with the forward and reverse geostrophic drag-law forms, Eqs. (3) and (5), one can write the predicted wind speed $U_{\text {pred }}$ in terms of the prediction-site roughness $z_{0,2}$ and geostrophic wind $G$. The geostrophic wind is further expressible in terms of the measured wind $U_{\mathrm{obs}}$, measurement height $z_{\mathrm{obs}}$, and background roughness $z_{0,1}$ for the measurement site. The resulting expression for $U_{\text {pred }}$ can be differentiated with respect to any of $\left\{U_{\mathrm{obs}}, z_{\mathrm{obs}}, z_{\text {pred }}, z_{0,1}, z_{0,2}\right\}$ in order to find the sensitivity of predicted wind speed $U_{\text {pred }}$ to these quantities. We would like to know the effect of roughness uncertainty upon $U_{\text {pred }}$; taking its derivative with regard to the roughness lengths at observation and prediction heights and rearranging, we obtain the useful expressions 


$$
\begin{aligned}
& \frac{\partial \ln U_{\text {pred }}}{\partial \ln z_{0,1}} \simeq \frac{1}{\ln \left(z_{\mathrm{obs}} / z_{0,1}\right)}\left[1-\frac{1}{\ln \left[G /\left(f z_{0,2}\right)\right]-A_{0}}\right] \\
& \times\left\{1-\left[\frac{U_{\mathrm{obs}} / G}{\ln \left(z_{\mathrm{obs}} / z_{0,1}\right)}\right]^{2}\left[\ln \left(\frac{\kappa U_{\mathrm{obs}}}{f z_{0,1}}\right)-A_{0}\right]\left[\ln \left(\frac{z_{\mathrm{obs}}}{z_{0,1}}\right)-1\right]\right\}
\end{aligned}
$$

and

$$
\frac{\partial U_{\text {pred }}}{\partial \ln z_{0,2}} \simeq\left(\frac{c_{G} G}{\kappa}\right) \frac{A_{0}+\ln \left(z_{\text {pred }} f / G\right)}{\left[\ln \left(G /\left(f z_{0,2}\right)\right)-A_{0}\right]^{2}} .
$$

Here we have made the expression compact by writing $G\left(U_{\mathrm{obs}}, z_{\mathrm{obs}}, z_{0,1}\right)$ simply as $G$. Inspection of the two sensitivity expressions, Eqs. (6) and (7), reveals that $U_{\text {pred }}$ is more sensitive to the background roughness at the observation site $\left(z_{0,1}\right)$ than the roughness $z_{0,2}$ at the prediction site. Furthermore, it is seen that $U_{\text {pred }}$ also has some sensitivity to observation height $z_{\mathrm{obs}}$, while $z_{0,1}$ dominates.

From Eqs. (A1)-(A2), which follow from Eqs. (6)-(7) (see Appendix A for details), we arrive at an (implicit) expression relating the uncertainty in predicted hub-height wind speed to the uncertainty in background roughness at the observation site $\left(\Delta z_{0,1}\right)$ :

$$
\begin{aligned}
\left.\frac{\Delta U_{\text {pred }}}{U_{\text {pred }}}\right|_{\Delta z_{0,1}} & \simeq \exp \left\{1.1\left(1+\frac{z_{\text {obs }}}{80 \mathrm{~m}}\right)^{-1 / 7}\right. \\
& \left.\times\left[\operatorname{li}\left\{\left(z_{\text {obs }} / a_{1} z_{0,1}\right)^{-1 / 7}\right\}-\operatorname{li}\left\{\left(z_{1} / z_{0,1}\right)^{-1 / 7}\right\}\right]\right\}
\end{aligned}
$$

where $\operatorname{li}(x)$ is the log-integral function (e.g., Abramowitz and Stegun, 1972; see appendix also). In Eq. (8), $a_{1}$ is the fractional uncertainty in observation-site background roughness length,

$a \equiv \frac{z_{0}+\Delta z_{0}}{z_{0}}=1+\Delta z_{0} / z_{0}$,

evaluated at $z_{0}=z_{0,1}$. Thus, roughness uncertainties can be described geometrically (as they should be): for a given background roughness, we then have a range of log roughness described by $\ln \left(z_{0,1}\right) \pm \ln (a)$ and corresponding roughness lengths ranging from $z_{0,1} / a$ to $a z_{0,1}$.

Just as Eq. (8) was derived above for variations in roughness at the measurement site, we similarly derive the uncertainty in predicted wind speed due to uncertainty in the prediction-site roughness $z_{0,2}$ from Eq. (7):

$$
\left.\frac{\Delta U_{\text {pred }}}{U_{\text {pred }}}\right|_{\Delta z_{0,2}} \simeq \frac{\left[1-\frac{\ln a}{\ln \left(z_{\text {pred }} / z_{0,2}\right)}\right]}{\left[1+\frac{\ln a}{A-\ln \left[G /\left(f z_{0,2}\right)\right]}\right]} .
$$

This follows from Eq. (A3), which includes details of the derivation (Appendix A).

The sensitivity of hub-height (predicted) wind speed to $z_{0,1}$, via Eq. (8), is shown in Fig. 3 for the case of $z_{\text {obs }}=60 \mathrm{~m}$ observation height and a hub height of $100 \mathrm{~m}$. Similarly, the uncertainty in predicted wind speed due to uncertainty in prediction-site roughness $z_{0,2}$, via Eq. (10), is displayed in Fig. 4.

The estimated relative uncertainty in predicted wind speed $\left(\Delta U_{\text {pred }}\right)$ is first plotted vs. fractional roughness uncertainty $a$ for a number of different measurement-site background roughnesses $\left(z_{0, \text { mast }}\right)$, and then it is also plotted against $z_{0 \text {,mast }}$ for different relative roughness uncertainty $\left(\Delta z_{0, \text { mast }} / z_{0, \text { mast }}=a-1\right)$, expressed as a percentage. For small background roughnesses one can see less effect on predicted wind speed for a given roughness error or uncertainty, with a nearly linear dependence of relative wind speed uncertainty upon $z_{0 \text {,mast }}$ for measurements taken over smooth land or water $\left(z_{0 \text {,mast }}<\sim 1 \mathrm{~cm}\right)$. For larger magnitudes of roughness uncertainty, as expected, one sees larger expected uncertainty in wind speed as well; this effect is reduced for smooth measurement sites (in conjunction with the previous statement). Also, for higher background roughnesses, the sensitivity of wind speed to (relative) roughness error is amplified, as shown by the green lines in panel a or the right-most (high $z_{0}$ ) part of panel (b) in Figs. 3-4. Comparing Figs. 34 , one also sees that the effect of a given change (or uncertainty in) $z_{0,2}$ has the opposite sign of the corresponding effect due to an equal change in $z_{0,1}$, but with the measurement or mast location's roughness $z_{0,1}$ having a larger effect than the prediction site roughness $z_{0,2}$. That is, the magnitudes of $\Delta U_{\text {pred }}\left(\Delta z_{0,1}\right)$ in Fig. 3 are larger than the magnitudes of $\Delta U_{\text {pred }}\left(\Delta z_{0,2}\right)$ displayed in Fig. 4.

Roughness bias and combined effect of $z_{0}$ sensitivities at measurement and prediction sites

Above we saw that wind speeds predicted via the GDL (Eq. 3) with roughness-affected (logarithmic) wind profile (Eq. 1) can be more sensitive to $z_{0,1}$ than to $z_{0,2}$. Thus, for an overall bias in roughness estimates, we should expect a net bias in wind speed predictions via wind atlas methods. In other words, for roughnesses that are systematically overestimated (or underestimated) by the same factor $a_{\text {bias }}$ at measurement and prediction sites, we then expect a corresponding bias in predicted mean wind speed. This effect is shown by Fig. 5, which displays the fractional change in predicted wind speed as a function of fractional change in measurement and prediction-site $z_{0}$ for combinations of $\left\{z_{0,1}, z_{0,2}\right\}$ that span typical application (colored lines).

As one might expect, for measurement and observation sites with similar background roughness, the change $\Delta U_{\text {pred }} / U_{\text {pred }}$ is relatively small, especially for systematically underestimated roughness lengths $\left(a_{\text {bias }}<1\right)$. Figure 5 also shows that for small biases $\left(a_{\text {bias }} \rightarrow 1\right)$, the wind speed prediction error is larger when the roughnesses at measurement and prediction sites are dissimilar. However, for roughness errors of a factor of $\sim 2$ or more, the nonlinearity of Eq. (3) with (1) complicates the dependence of $\Delta U_{\text {pred }}$ on 

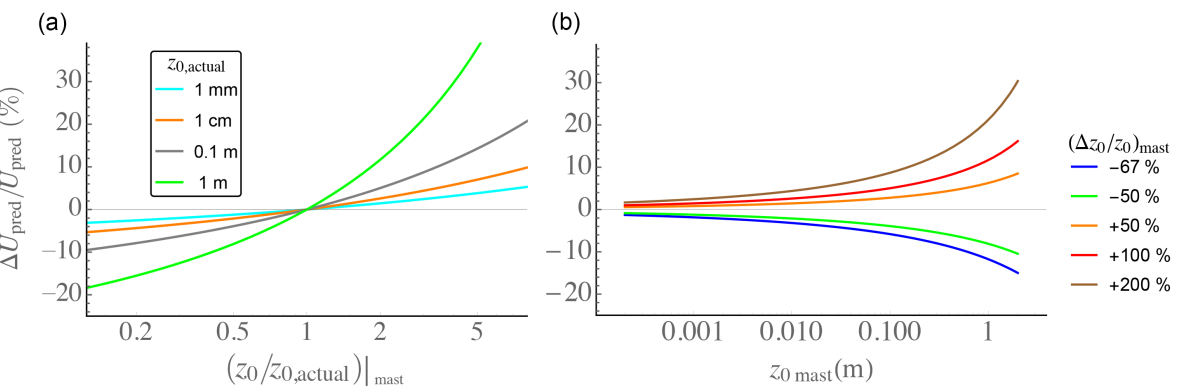

Figure 3. Error in predicted wind speed due to error in background roughness at measurement site via Eq. (8), for observation height $z_{\mathrm{obs}}=60 \mathrm{~m}$ and prediction (hub) height of $z_{\text {pred }}=100 \mathrm{~m}$. (a) Error vs. ratio $(=a)$ of estimated to actual background $z_{0}$. (b) Error vs. background $z_{0}$ at observation mast; uncertainties of $\{-67,-50,50,100,200\}$ correspond to $a=\left\{\frac{1}{3}, \frac{1}{2}, 1.5,2,3\right\}$.
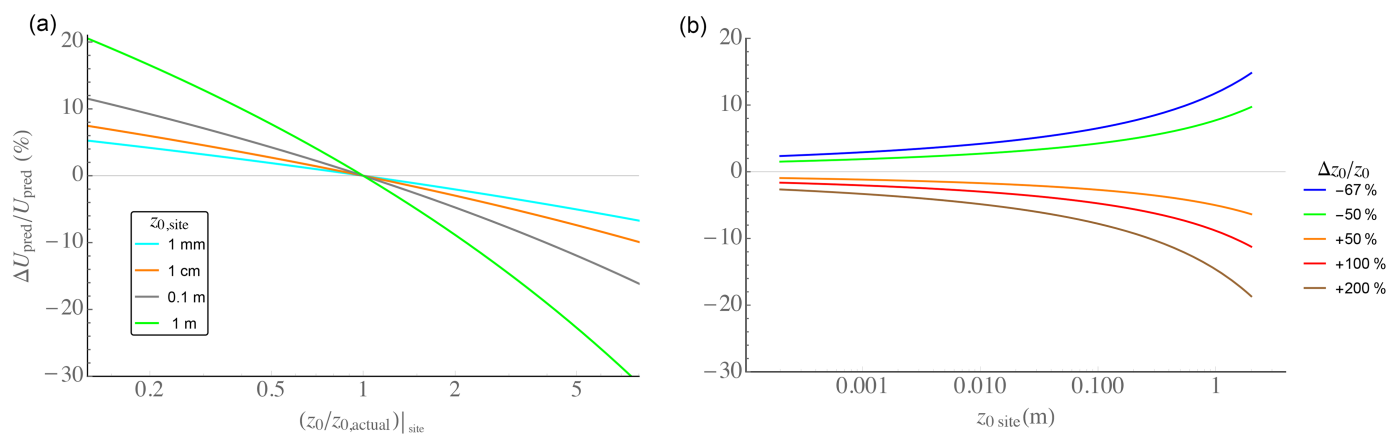

Figure 4. Error in predicted wind speed due to error in background roughness at prediction site via Eq. (10), for observation height $z_{\mathrm{obs}}=60 \mathrm{~m}$ and prediction (hub) height of $z_{\text {pred }}=100 \mathrm{~m}$. (a) Error vs. ratio $(=a)$ of estimated to actual background $z_{0}$. (b) Error vs. background $z_{0}$ at observation mast; uncertainties of $\{-67,-50,50,100,200\}$ correspond to $a=\left\{\frac{1}{3}, \frac{1}{2}, 1.5,2,3\right\}$.

$a_{\text {bias. }}$ In addition to the typical range of $z_{0}$ used in wind resource estimation (colored lines), Figure 5 also shows the gross effect of measurement over forest (or effectively more complex terrain, i.e., with effective roughness $z_{0,1}=1 \mathrm{~m}$; denoted by grey lines); one can see the corresponding increase in $\Delta U_{\text {pred }} / U_{\text {pred }}$ for such cases when there is overestimation of $z_{0}$, even if $z_{0,2}$ and $z_{0,1}$ are both $1 \mathrm{~m}$.

In contrast to a possible bias in roughness assignment, one can imagine a worst case scenario as having a negative error in $z_{0,1}$ and a positive error in $z_{0,2}$ (or vice versa), e.g., $a_{1}=1 / a_{2}$. In this scenario the result resembles the plots in Fig. 5, but rotated $45^{\circ}$ with the $y$ axis stretched by a factor of 2: cases with $z_{0,1}=z_{0,2}$ no longer have small error, but all the lines show a large uncertainty for $a$ far from 1 (e.g., $\pm 40 \%$ at $a_{1}^{ \pm 1}=a_{2}^{\mp 1}=0.1$ for $z_{0,1}=1 \mathrm{~cm}$ and $z_{0,2}=1 \mathrm{~m}$, corresponding to the solid green line), and all lines have $\Delta U_{\text {pred }}=0$ for $a=1$.

A more general situation is that of independent errors in roughness assignment at different sites. In this limit, one foresees a distribution of $\Delta U_{\text {pred }}$, given uncertainties in $z_{0,2}$ and $z_{0,1}$ (basically $P\left(z_{0,2}\right)$ and $P\left(z_{0,1}\right)$ ). Two examples of this are given in Fig. 6 . The figure shows $P\left(\Delta U_{\text {pred }} / U_{\text {pred }}\right)$ for the cases of winds observed over grass but predicting winds over grass or forest, where the grass and forest $z_{0}$ have log-normal distributions $P(a)$ with means and widths given for the combined samples in Table 1. Following the earlier examples, the observation height is taken as $60 \mathrm{~m}$ and prediction (hub) height is $100 \mathrm{~m}$.

In the figure, one can see the combined effect of different roughness distributions and uncertainties, particularly for the case of grass to forest (panel b in Fig. 6). For this case, the half width of the grass $P\left(z_{0,1}\right)$ corresponds to $117 \%$ (where $\left.\left\langle z_{0,1}\right\rangle=4 \mathrm{~cm}\right)$ and that for the forest corresponds to $141 \%$ of $\left\langle z_{0,2}\right\rangle=0.85 \mathrm{~m}$ following Table 1 . The combined effect gives wider error distributions $P\left(\Delta U_{\text {pred }}\right)$ for the grass-toforest case than for the grass-grass case, as expected from Fig. 5, for example; the standard deviations corresponding to the $z_{0}$-induced mean-wind error distributions in Fig. 6 are 1 and $4 \%$ for the predictions over grass and forest, respectively (and both error distributions are nearly Gaussian, with skewnesses of 0.02 and -0.2 ). To be yet more conservative, if we follow Sect. 2.1.2 using a gross estimate of observational $z_{0}$ uncertainty equivalent to a half width (roughness uncertainty factor) of $w_{\left\langle z_{0}\right\rangle_{\mathrm{RS}}} \sim 3$, the uncertainty $\sigma_{\Delta U_{\text {pred }} / U_{\text {pred }}}$ (distribution widths) for the two cases shown in Fig. 6 grow to 8.6 and $14 \%$. Towards practical considera- 


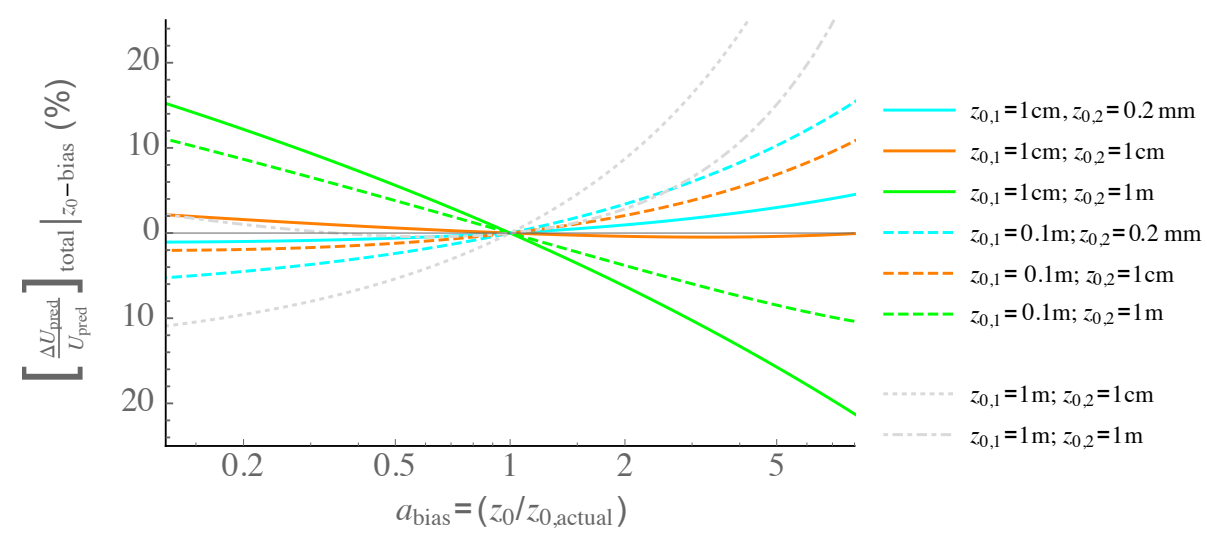

Figure 5. Total error in predicted wind speed due to a bias $\left(a_{\text {bias }}\right)$ in background roughness at both prediction and measurement sites for different combinations of background roughness at the sites. As in Figs. 3-4, observation height is $z_{\mathrm{obs}}=60 \mathrm{~m}$ and prediction (hub) height is $z_{\text {pred }}=100 \mathrm{~m}$.
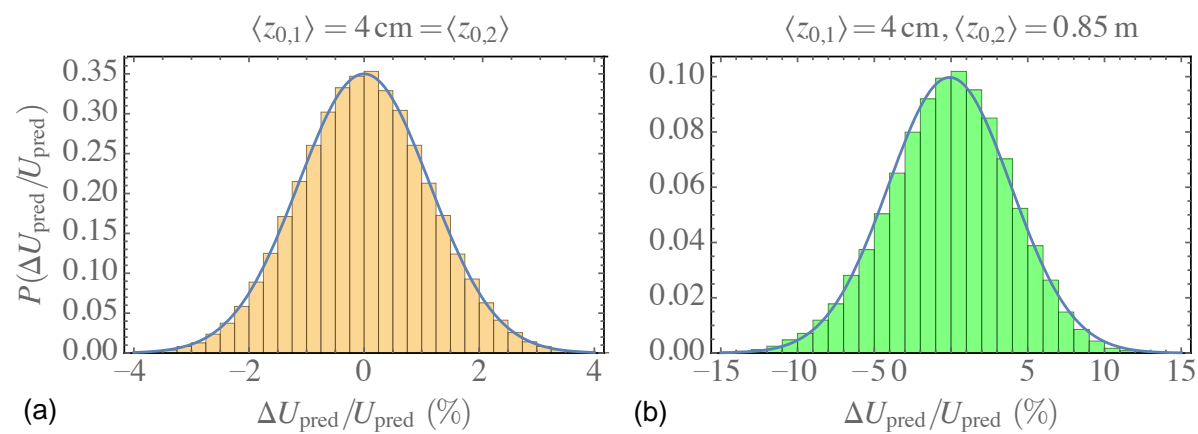

Figure 6. Distribution of error in predicted wind speed, given distributions $P\left(z_{0,2} /\left\langle z_{0,2}\right\rangle\right)$, and $P\left(z_{0,1} /\left\langle z_{0,1}\right\rangle\right)$ at prediction and measurement sites. (a) Prediction from grass to grass and (b) from grass to forest. Input $P\left(z_{0}\right)$ follows from elicited samples in Table 1; see text. Blue line is normal distribution based on calculated mean and standard deviation. As in Figs. $3-5, z_{\mathrm{obs}}=60$ and $z_{\text {pred }}=100 \mathrm{~m}$.

tion for wind engineers, we also point out that for prediction over water (again from $z_{\text {obs }}=60$ to $z_{\text {pred }}=100 \mathrm{~m}$ with $z_{0,1}=4.1 \mathrm{~cm}$ ) using the conservative roughness-uncertainty estimate $a=w_{\left\langle z_{0}\right\rangle_{\mathrm{RS}}} \sim 3$ again leads to uncertainty in $U_{\text {pred }}$ that exceeds $6 \%$ and an error distribution that is somewhat non-Gaussian (skewness $\approx 0.6$, plot not shown); we provide this number to demonstrate the roughness-induced uncertainty expected when using land-based measurements for offshore predictions.

\subsubsection{Sensitivity of predicted energy production to background $z_{0}$}

The uncertainty in background roughness can also be translated into AEP uncertainty by employing a relation between wind speed and AEP, i.e., via a turbine (or perhaps wind farm) power curve. The propagation of $z_{0}$ uncertainty to AEP follows that derived for wind speed above, but with some assumptions. First, we assume Weibull-distributed winds, which is standard practice in wind energy and also facilitates analytical derivation of a bulk relation between AEP and mean wind speed $\langle U\rangle$. Because power curves in practice do not have a "kink" at rated wind speed, but rather a smooth transition from the ideal $\langle U\rangle^{3}$ regime to the maximum (rated) power regime of operation (e.g., Wagner et al., 2011), we can derive an analytical effective power-curve form, expressible as a function of $\langle U\rangle / V_{\text {rat }}$, i.e., Eq. (B1) (shown in Appendix B). To accomplish analytical integration and readily relate mean wind speed (or Weibull- $A$ parameter) per turbine-rated speed, some mathematical approximations are used, wherein we also assume that the Weibull- $k$ parameter is close to a value of 2 (within 10$20 \%)$. The analytical power-curve form $\operatorname{PC}\left(U / V_{\text {rat }}\right)$ then leads to a power-law relation between normalized AEP and wind speed: $\mathrm{AEP}_{\text {norm }} \propto\left(\langle U\rangle / V_{\text {rat }}\right)^{p}$, where the power-law exponent $p$ is also a function of $\langle U\rangle / V_{\text {rat }}$, as shown in Appendix B.

Figure 7 shows an example of AEP sensitivity to fractional roughness uncertainty of the observation site (ratio of estimated to actual $z_{0,1}$, i.e., $a=\Delta z_{0,1} / z_{0,1}+1$ as in Eq. 9) for the case of $\langle U\rangle=0.7 V_{\text {rat }}$; the latter translates to a power exponent of $p \simeq 1.85$ for the analytical power-curve form elu- 


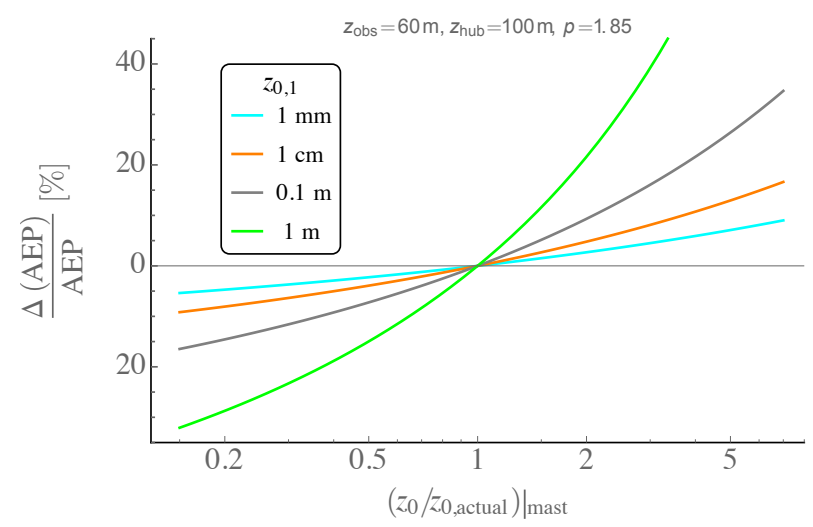

Figure 7. Sensitivity of (error in) predicted normalized power due solely to error in background roughness at measurement site vs. ratio of estimated to actual background $z_{0}$ at observation mast (i.e., $1+$ relative error, Eq. 9) for various values of actual $z_{0,1}$. Observation height is $60 \mathrm{~m}$ and hub height is $100 \mathrm{~m}$, as in Figs. 3-6.

cidated in Appendix B (see Fig. A4b). For Fig. 7 we consider the same situation as used for Figs. $3-5\left(z_{\mathrm{obs}}=60\right.$ and $z_{\text {pred }}=100 \mathrm{~m}$ ). As one might expect, the AEP uncertainty - due to uncertainties in $z_{0,1}, z_{0,2}$, or their combined effect with a common bias - simply resembles the wind speed uncertainty plots shown in Figs. 3-5: the vertical axis of the plot appears stretched by a factor of $p(\simeq 1.85)$. An analogous plot of the distribution of AEP error follows similarly; for a given value of $p$ (here 1.85), the horizontal ( $x$-) axes in the plots of Fig. 6 are stretched by a factor of $p$ to give the distribution of $\triangle \mathrm{AEP}$.

\subsection{Effect of uncertainty in background roughness upon wind resource predictions}

In order to give examples (and realistic numbers) useful to wind engineers, in this section we translate the observationbased (Sect. 3.1.1) and user-based (Sect. 3.1.2) roughness uncertainties into uncertainties of predicted mean wind speed and AEP for the observation and user-survey examples treated in Sect. 3.1.1 and 3.1.2, respectively.

\subsubsection{Uncertainty in predicted mean wind speeds}

The relative uncertainties implied by roughness lengths calculated via surface-layer wind speed measurements were outlined in Sect. 3.1.1 for the seemingly ideal grassy terrain east of Høvsøre. The half widths of the roughness distributions for the homogeneous sectors were found to be on the order of a factor of 3 times $\left\langle z_{0}\right\rangle$, while the uncertainty in obtaining a mean (representative) roughness was found through bootstrap resampling to be much smaller, about $5 \%$; this result came whether $z_{0}$ was calculated from speeds at multiple heights in the ASL or from sonic anemometer measurements of $U$ and $u_{*}$ in the ASL. However, despite similar dis- tribution widths and similar apparent uncertainty in meanestimation, the $\left\langle z_{0}\right\rangle$ themselves differed by roughly one-half order of magnitude, i.e., a factor of $\sim 3$ when determined in these two different ways. Thus, we first consider (conservatively) a relative uncertainty of $a \sim 3^{ \pm 1}$ for $z_{0}$, for the typical resource-assessment heights $\left(z_{\mathrm{obs}}=60, z_{\text {pred }}=100 \mathrm{~m}\right)$ used in Figs. 3-7. As seen in Fig. 5, for systematic (bias) overestimates of $a \equiv \Delta z_{0} / z_{0}$ and a mean roughness length at the observation site of $1 \mathrm{~cm}$, this translates into wind speed uncertainty values of less than $1 \%$ when predicting $100 \mathrm{~m}$ winds over the same roughness and gives $\Delta U_{\text {pred }}$ of $\{2,-2,-6,-10 \%\}$ for predictions over roughnesses of $z_{0,2}=\{0.2 \mathrm{~mm}, 3 \mathrm{~cm}, 30 \mathrm{~cm}, 1 \mathrm{~m}\}$. For the same magnitude of systematic underestimate $(a \sim 1 / 3)$ the corresponding $\Delta U_{\text {pred }} / U_{\text {pred }}$ are $\{-1,2,5,9 \%\}$ for these $z_{0,2}$, with an uncertainty of $1 \%$ for $100 \mathrm{~m}$ winds predicted over the same roughness as the measurement site. Thus, we see about $1 \%$ uncertainty in $U_{\text {pred }}$ for these typical heights and the same observation and prediction roughness. Meanwhile, using such observations to predict winds over nearby forested land, for example, incurs higher uncertainties, with magnitudes of 5$10 \%$, without yet considering modeling the flow over such terrain. To get estimates of $\Delta U\left(\Delta z_{0}\right)$ for other observation and prediction heights and roughnesses, we remind the reader that these can be obtained from Eqs. (8)-(10).

For the uncertainties inherent in user-provided roughness lengths, we address the two cases treated in Sect. 3.1.2. The grass case is similar to that considered in the Høvsøre analysis above, with a mean roughness of about $4 \mathrm{~cm}$. If we take the half width of the expected user-input distribution of $z_{0}$, i.e., $\exp \left\{\sigma_{\left.\ln \left[z_{0} / / z_{0}\right\rangle_{g}\right]}\right\}$ from Table 1, then we can again arrive at estimates for the wind-speed uncertainty (this is also a bit conservative because it gives larger uncertainties than the bootstrap-derived half width). Again assuming typical application heights $\left(z_{\text {obs }}=60, z_{\text {pred }}=100 \mathrm{~m}\right)$ for predictions over site roughnesses, $z_{0,2}=\{0.2 \mathrm{~mm}, 1 \mathrm{~cm}, 30 \mathrm{~cm}, 1 \mathrm{~m}\}$ and a $z_{0}$-bias of $2.2^{ \pm 1}( \pm 120 \%$ from Table 1$)$, we obtain $U_{\text {pred }}$ uncertainties of $+\{3,1,-3,-6 \%\}$ and $-\{2,0.4,-3,-5 \%\}$. These roughly correspond to (a proxy of) the industry-wide uncertainty in predicted wind speeds (with this $z_{\mathrm{obs}}, z_{\text {pred }}$ ) for observations over a background roughness like the grass in Fig. 2. For the surveyed forest roughness in that figure, we get corresponding $\Delta U_{\text {pred }}$ following Table 1 for the case of all-site biases $\left( \pm 141 \% \rightarrow a \approx 2.4^{ \pm 1}\right.$ applied to both $z_{0,1}$ and $z_{0,2}$. For predictions from observations over such a site, applied to turbine sites with $z_{0,2}=\{1 \mathrm{~cm}, 10 \mathrm{~cm}, 1 \mathrm{~m}\}$ we get $\Delta U_{\text {pred }} \approx(+)\{11,9,3 \%\}$ for systematic overestimates and $(-)\{6,4,0.3 \%\}$ for systematic $z_{0}$ underestimation. The latter finding is rather significant as it implies that an underestimation of forest roughness lengths is safer than overestimating $z_{0}$ when using EWA-based methods for wind resource estimates (e.g., WAsP and similar methods). This is consistent with common practice: while recent evidence from direct lidar scans of forests suggests that $z_{0}$ should be at least several 
meters there (Boudreault et al., 2015), industrial practice has been to use $z_{0}$ of $1 \mathrm{~m}$ or less (e.g., Troen and Petersen, 1989; Mortensen et al., 2014).

\subsubsection{Uncertainty in predicted energy production}

The magnitude of $z_{0}$-induced AEP uncertainty for typical simple sites depends in general on the ratio of $\langle U\rangle / V_{\text {rat }}$ (for classically behaved turbines) because the relationship between $\langle U\rangle$ and AEP depends on this ratio; this dependence is most simply expressed via the exponent

$p=\frac{\ln (\mathrm{AEP})}{\ln \langle U\rangle}$ for a power-law relation AEP $=\langle U\rangle^{p}$,

detailed in Appendix B. As mentioned in the previous section, with regards to uncertainty in the background roughness of either the observation or prediction site (or for a bias across both sites), the sensitivity plots of $\Delta\langle U\rangle$ per given roughness error are simply translated into analogous AEPsensitivity figures via stretching the vertical axes by a factor $p$ (as was done to get Fig. 7 from Fig. 3a); similarly the horizontal $(\Delta U)$ axis in Fig. 6 is stretched by a factor $p$. Since $p$ basically varies between $\sim 0.8$ and 2.5 (over the reasonable range of $\langle U\rangle / V_{\text {rat }} \sim 0.5-0.9$ ), then the mean wind speed uncertainties quoted in the previous subsection can be simply multiplied by a factor of $\sim 0.8-2.5$, depending on the expected turbine power curve and subsequent $p$.

For most general practical use, we ultimately consider roughness error distributions and the consequent AEP error distributions, such as those shown in Fig. 6. For independent roughness error distributions at measurement and prediction sites, and assuming log-normal distributed $\Delta z_{0}$ (as demonstrated in Sect. 3.1.1 and 3.1.2 for measured and userestimated distributions), via Eqs. (8) and (10) we can obtain distributions of $\triangle$ AEP. The uncertainty in $z_{0}$ can be expressed in terms of the dimensionless width $w_{z_{0}} /\left\langle z_{0}\right\rangle$; for a given width we can synthesize distributions of $z_{0,1}$ and $z_{0,2}$, and then find the standard deviation of the resulting distribution of $\triangle \mathrm{AEP}$. We do such Monte Carlo simulations over the range of dimensionless widths from 5 to $500 \%$ for the same $\left\{z_{0,1}, z_{0,2}\right\}$ pairs and observation and prediction heights as used in Fig. 5; the results are shown in Fig. 8.

Figure 8 shows AEP uncertainty vs. roughness uncertainty; the latter is expressed as the dimensionless width $w_{z_{0}} /\left\langle z_{0}\right\rangle$ of the $z_{0}$ distribution, calculated via the standard deviation of $\ln \left(z_{0} /\left\langle z_{0}\right\rangle\right)$ as in Eq. (4). Following the previous subsection's analysis (where $z_{\mathrm{obs}}=60 \mathrm{~m}, z_{\text {pred }}=100 \mathrm{~m}$, and $p=1.85$ ), Fig. 8 shows that for actual measurementsite roughness $z_{0,1} \approx 1-10 \mathrm{~cm}$, given a relative $z_{0}$ uncertainty of $100 \%$ (corresponding to $w_{z_{0}} \sim z_{0}$ as in Sect. 3.1.2), the GDL/ $z_{0}$-induced AEP uncertainty ranges from $\sim 5 \%$ (for $\left\langle z_{0,1}\right\rangle=1 \mathrm{~cm}$, predicting over water) to $15 \%$ (for $\left.\left\langle z_{0,1}\right\rangle=10 \mathrm{~cm},\left\langle z_{0,2}\right\rangle=1 \mathrm{~m}\right)$. For the statistical uncertainty example of (mostly) homogeneous flat farm and/or grassland shown previously in Fig. 1 (Sect. 2.1.2), taking the relative background roughness uncertainty factor to be equivalent to the width of the $\ln \left(z_{0}\right)$ distribution (centered around $\sim 1.4 \mathrm{~cm}$ for wind directions from $\sim 45$ to $120^{\circ}$ ), i.e., $a \sim 3^{ \pm 1}$, leads to a similar AEP uncertainty range, roughly $6-16 \%$ for prediction sites ranging from water to forest or urban. However, such an uncertainty estimate seems large and may be explained considering Table 2. For industrial use, wind engineers (e.g., in medium or large companies) in effect assign a kind of ensemble-average roughness length for any given land-use type. Consider, for example, the case of taking three community-accepted values for the grass site as in Table 2 , i.e., a relative $z_{0}$ uncertainty of roughly $50 \%$, one can see from Fig. 8 that the AEP uncertainty drops to $4-10 \%$. One is reminded that these AEP uncertainty values correspond to the case of observation and prediction heights of 60 and $100 \mathrm{~m}$, respectively: the slight dependence of $\triangle \mathrm{AEP}$ on $z_{\mathrm{obs}}$ and $z_{\text {pred }}$ modifies the uncertainty for other heights. Aside from the weak dependence on measurement and prediction heights, one also sees a basic power-law form emerging for the AEP estimates:

$\frac{\sigma_{\mathrm{AEP}}}{\langle\mathrm{AEP}\rangle} \propto \sim\left(\frac{w_{z_{0}}}{\left\langle z_{0}\right\rangle}\right)^{6 / 7}$,

particularly for relative roughness uncertainties (widths of the distribution $\left.P\left(\ln z_{0} / \ln \left\langle z_{0}\right\rangle\right)\right)$ that are

$w_{z_{0}} /\left\langle z_{0}\right\rangle<\sim 1-2$.

We also note again that we have focused here on the AEP uncertainty caused by uncertainty in background roughness rather than the $z_{0}$ uncertainty itself. Further details of the latter are the subject of ongoing work and another paper, and here we point to Fig. 8 as the significant result: for a given uncertainty in $z_{0}$, one can find the corresponding uncertainty in AEP due to use of the GDL-EWA method.

\section{Conclusions}

First we review the context of this work, i.e., the EWA method (Troen and Petersen, 1989) ${ }^{5}$, which employs the geostrophic drag law (Eq. 3) to perform horizontal extrapolation: mean wind speed measured at a site with some background roughness(es) can be used to predict the mean wind at another location with potentially different surface characteristics, assuming the sites are forced by the same pressure gradient (geostrophic wind). For separate measurement and/or prediction sites where the EWA method is valid ${ }^{6}$, resource

\footnotetext{
${ }^{5}$ The EWA method is implemented in WAsP and related software (e.g., windPRO, WindFarmer).

${ }^{6}$ The GDL applies to sites with approximately the same latitude and geostrophic-scale forcing (roughly the distribution of geostrophic wind); the scale of spatial variations in the geostrophic wind depends on the terrain complexity and can vary from several tens of kilometers in simple terrain down to just a few kilometers in very complex terrain or near coasts; see Troen et al. (2014), Hahmann et al. (2015).
} 


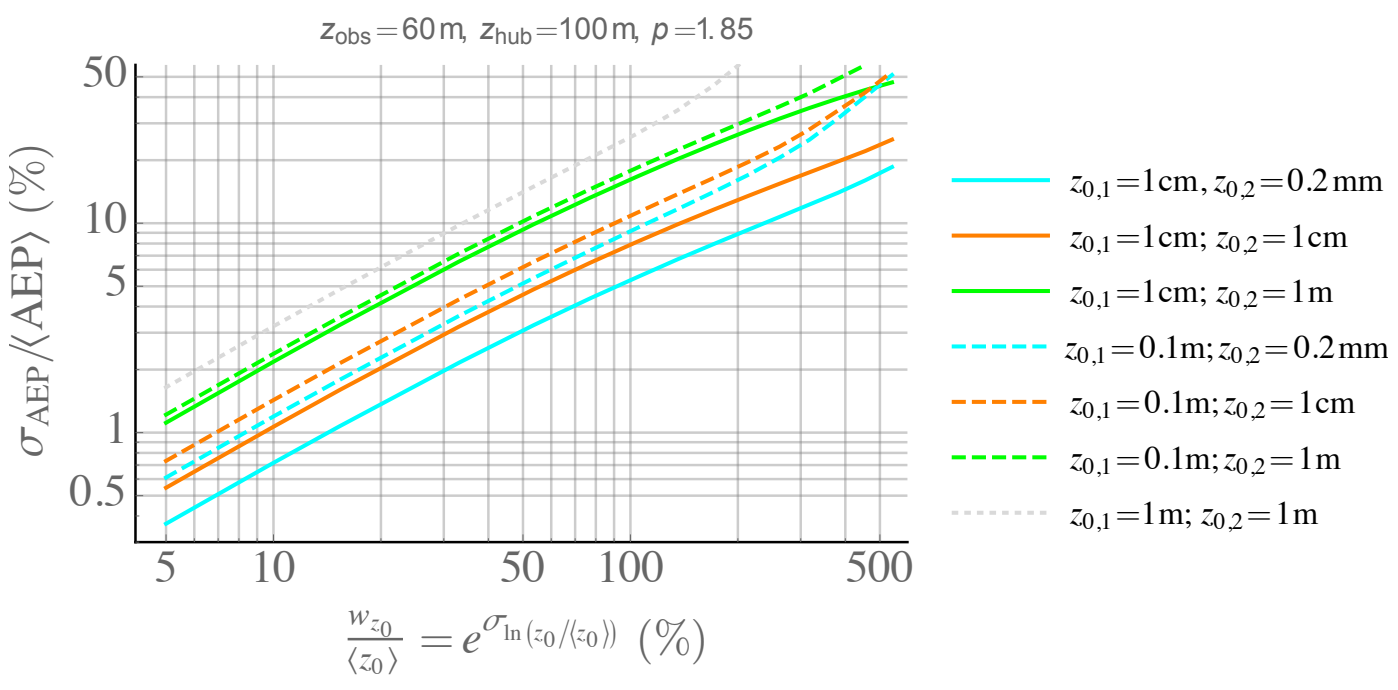

Figure 8. Uncertainty in AEP vs. (relative) roughness uncertainty due to the combined effect of observation and prediction site roughnessuncertainty; independent log-normal roughness distributions assumed, with dimensionless width $w_{z_{0}} /\left\langle z_{0}\right\rangle$. Standard deviation of AEP shown for different combinations of $\left\langle z_{0,1}\right\rangle$ and $\left\langle z_{0,2}\right\rangle$. Case shown for $p=1.85$, i.e., AEP $=U^{1.85}$ as in Fig. 7.

assessments that account for background roughness length $\left(z_{0}\right)$ tend to be better than assessments that ignore $z_{0}$ (such as those based only on observed shear exponent; see Kelly, 2016). This is especially true for sites in terrain with different background roughness; consequently, the EWA method has been used in wind energy for decades. The need for and justification of this method is also implied by Fig. 4, which displays the sensitivity of EWA-predicted winds to turbinesite roughness $\left(z_{0,2}\right)$; it can thus also be used to show how much the predicted mean wind changes due to $z_{0,2}$ differing from the measurement-site roughness $z_{0,1}$. For $z_{0,2} / z_{0,1}$ deviating significantly from 1 (taking the $x$ axis of Fig. $4 \mathrm{a}$ as this ratio), a significant $\Delta U_{\text {pred }}$ can result, and the EWA method is needed to account for such. One can see that if $z_{0,2}$ differs from $z_{0,1}$ by a factor of 5 , the predicted mean wind may be affected by $\sim 5-25 \%$; subsequently, the AEP could change by a factor of up to $\sim 2.5$ times this, i.e., as much as $\sim 60 \%$.

Using the EWA method, uncertainty in $z_{0}$ leads to uncertainty in resource predictions that can be significant, as shown in Sect. 3. Both user-implicit (Sect. 3.1.2) and definition-related (Sect. 3.1.1) uncertainties in roughness length are found to effectively be (treatable as) roughly of the same order of magnitude, and they lead to an uncertainty in prediction of mean wind speed and AEP. The uncertainty in prediction is slightly more sensitive to measurement-site roughness $z_{0,1}$ than prediction-site roughness $z_{0,2}$, as seen in Eqs. (8)-(10) and displayed in Figs. 3-4. However, there is also a minor dependence on measurement and prediction heights via the vertical wind profile used within the EWA method (log law implicit in Eqs. 8, 10); shown by Fig. A2 in Appendix A.
As mentioned in Sect. 3.1.1, even in ideal (steady, neutral) conditions, the mean roughness $\left\langle z_{0}\right\rangle$ obtained from observations and Eq. (1) via different calculation methods in the surface layer, such as using wind speeds at multiple heights or alternately wind speed with friction velocity, differs by an amount that appears to greatly exceed the uncertainty derived for any given method. For example, bootstrapped distributions of $\left\langle z_{0}\right\rangle$ for the homogeneous flat grassland sectors at Høvsøre had relative widths (approximate uncertainty) well under $10 \%$ when using Eq. (1) and $U_{\text {obs }}$ in the surface layer, whether calculated with or without $u_{*}$; however, the ratio of the means (or peaks of $P\left(\left\langle z_{0}\right\rangle\right)$ ) from the different calculation methods was roughly 3 . In contrast, the uncertainty of $z_{0}$ estimated from polls of two groups of wind resource assessment experts (for grassland and forest) in Sect. 3.1.2 was on the order of $z_{0}$ itself, i.e., $w /\left\langle z_{0}\right\rangle \sim 1$ when estimated from single values of $z_{0}$ as in Table 1 ; such uncertainty shrinks, however, if assuming that wind engineers gauge roughness from a collection of accepted sources, as in the example of Table 2.

We note that more exact quantification of measured roughness uncertainty involves consideration of numerous other factors, from ABL physics and fluid dynamics to inhomogeneous boundary conditions and turbulent transport. Likewise, more accurate characterization of epistemic user-based (industry-wide) uncertainty would likely require a much wider survey for a greater number of roughnesses. Here we have made a basic evaluation of the main roughness uncertainty components and their approximate magnitudes, focusing first on what resultant uncertainty can be expected in a wind resource prediction, given some level of roughness uncertainty. The latter focus leads to analysis culminating in Fig. 8 , which visualizes a primary result of this work: the 
uncertainty in AEP (or scaled mean wind) predicted via the EWA method for a given uncertainty in background roughness length and pair of surface types (roughnesses) at separate prediction and measurement sites. From Fig. 8 we see that the basic trend for uncertainty in mean wind speed or AEP behaves as approximately $\left(w /\left\langle z_{0}\right\rangle\right)^{6 / 7}$ in the dimensionless roughness uncertainty regime $w /\left\langle z_{0}\right\rangle<\sim 200 \%$, i.e., just within the range we have estimated.

There are other sources of uncertainty implicit in the use of the EWA method, in addition to the roughness lengths. Additional uncertainties include the applicability of the GDL (see footnote 6$)$, the constants $(A, B)$ within Eq. (3), and the actual form and/or use of Eq. (3) with arguments averaged in an ensemble (or spatial) sense. These are beyond the scope of the current paper. However, as for applicability of the GDL, regarding the distance between measurement and prediction sites, we remind the reader that (fine-resolution) mesoscale models give an indication of the spatial extent (and direction) of variations in the geostrophic wind, and we refer the reader to Hahmann et al. (2015) and Troen and Petersen (1989), for example. As to the distance over which one may horizontally extrapolate in more complex terrain, this depends upon the observation and prediction heights, along with the terrain complexity (as ruggedness index RIX, Mortensen et al., 2006, or local elevation variability Kelly et al., 2014a, Kelly, 2016); we point the reader to Clerc et al. (2012) and Troen et al. (2014) for uncertainty in complex terrain. The minor uncertainties due to GDL constants $(A, B)$ are the subject of ongoing work (e.g., Floors et al., 2015), and the GDL averaging issue is currently seen to be secondary due to the wellbehaved nature of Eqs. (8) and (10) and the magnitude of $z_{0}$ variations expected.

Additional uncertainties can also arise due to the use of a (mean) wind profile expression, such as the simple log law (Eq. 1) invoked here. One uncertainty is due to the applicability of a given profile model. Following Troen and Petersen (1989) and due to the statistical dominance of neutral conditions (Kelly and Gryning, 2010), we have used the (surface-layer) form (Eq. 1) applicable in neutral conditions; furthermore, we limit our observational analysis to neutral steady conditions and observations to be within the surfacelayer, where the logarithmic profile is valid and the roughness length is simply defined. However, deviations from logarithmic may occur above the surface layer, such as for the prediction height considered in the figures $(100 \mathrm{~m})$, in the case of very shallow ABL depths (i.e., depths less than $\sim 2 z_{\text {pred, }}$, Pedersen et al., 2014, or $200 \mathrm{~m}$ in this case) that occasionally occur (Liu and Liang, 2010). This ABL-depth effect is negligible for $z_{\text {pred }}$ close to $z_{\text {obs }}$ (and $z_{0,1} / z_{0,2}$ near 1 ) and is minor for the heights considered. However, an additional uncertainty dependent upon the ABL depth could be modeled following Kelly and Gryning (2010) and Liu and Liang (2010), or alternately a better profile form (e.g., Kelly and Gryning, 2010) could be invoked along with the GDL, particularly to reduce uncertainties for predictions well above
$100 \mathrm{~m}$ or in areas where lower-level jets are expected. Another uncertainty arising implicitly from the profile model, as analyzed here, is due to considering the same $z_{0}$ for use in both the profile model and the GDL. That is, the wind profile reacts to a more local roughness, whereas the GDL reacts to a geostrophic-scale $z_{0}$. In Troen and Petersen (1989) the latter is obtained by taking a weighted geometric spatial average of $z_{0}$, where $\ln z_{0}$ is integrated upwind from a given location with a weighting function that decays with distance ${ }^{7}$; thus, the local and geostrophic $z_{0}$ can differ slightly. This is not likely to have a major effect on the analysis here since the Høvsøre sectors considered were ideal and without significant inhomogeneity, such that the upwind-averaged roughness is within $10 \%$ of the local $z_{0}$. However, it is worth noting that for large roughness changes (e.g., coastlines) within $\sim 10 \mathrm{~km}$ upwind of a site, the geostrophic $z_{0}$ will differ from the site's $z_{0}$; Eqs. (8)-(10) can be recast for such. The effect on roughness uncertainty incurred through such spatial averaging is expected to be (much) smaller than the crude factor $w /\left\langle z_{0}\right\rangle \sim 3(200 \%)$ found and presented above, though systematic evaluation of this effect is still a subject of ongoing research. Analogously, the height-dependent effect of inhomogeneities upon roughness (i.e., above the ASL) - in particular its uncertainty - is also under study, but is expected to be minor for simple terrain.

Vertical extrapolation has not been treated explicitly here, though it is implicit in the vertical profile used to estimate $u_{*}$ from observed wind for use in the GDL. Such treatment, in conjunction with taking the profile roughness and geostrophic-scale roughness to be the same, is a choice that we have made to facilitate systematic modeling of roughness-induced uncertainty; thus, we have been able to estimate the effect of roughness, which occurs through both the wind profile (vertical extrapolation) and through invocation of the GDL (horizontal extrapolation). A separate model for the uncertainty in vertical extrapolation using a logarithmic-based profile (as in the EWA and popular wind software, e.g., WAsP), but without considering roughness uncertainty, is given in Kelly and Troen (2016) and Kelly (2016). Treating the $z_{0}$-related uncertainties separately, per the geostrophic drag law and wind profile, is the subject of continuing work beyond the scope of the current article.

\subsection{Applications and implications}

In increasingly complex terrain, the actual surface roughness becomes less significant compared to terrain slope with regards to affecting the flow. However, for horizontal extrapolation, the aggregate effect of the (complex) terraininduced drag leads to an increase in the effective geostrophic-

\footnotetext{
${ }^{7}$ The EWA roughness-averaging weighting function is prescribed as $\exp \left(-r / \ell_{r}\right)$, where $r$ is the distance upwind, $\ell_{r}$ is a length scale generally taken to be $10 \mathrm{~km}$ (as default WAsP value), and the integration is carried out to $20-30 \mathrm{~km}$ (roughly half the Rossby radius).
} 
scale roughness (Beljaars et al., 2004; Kelly et al., 2014a). Thus, the geostrophic-drag and roughness uncertainty analysis given in this work can also be applied towards improved use of microscale models in complex terrain when horizontal extrapolation is involved. In particular, computational fluid dynamics solvers (e.g., RANS and LES), when employed using different simulation domains for measurement and wind farm sites, are typically used to calculate terrain-induced flow perturbations (speed-up factors) at the respective sites. However, for domains with different degrees of complexity (or potentially different resolutions) - and thus different largescale drag - then the use of the geostrophic drag law (or any analogous empirical algorithm or method) demands that measured wind statistics must additionally be transformed properly, accounting for differences in the effective domainscale mean roughness in the two domains (per wind direction). Thus, uncertainty in characterizing the effective roughness due to terrain drag can be translated into a corresponding uncertainty in mean wind (or AEP) via the framework presented here. Alternately, for a given pair of (observation, prediction) sites, the uncertainty in mean wind prediction due to neglect of terrain drag can be estimated: a bias is introduced, whereby the effective geostrophic roughness is underestimated. From Fig. 5 one can see, for example, that for sites with the same effective roughness (complexity) of $z_{0, \text { eff }} \sim 1 \mathrm{~m}$ and with an underestimation of 1 order of magnitude $\left(a_{\text {bias }} \simeq 0.1\right)$, a positive error $\Delta U_{\text {pred }} \sim 2 \%$ is incurred.

Another implication of this work applies to assessment in forested regions. Some work on characterizing profileamenable roughness over forest (e.g., Bosveld, 1997; Tian et al., 2011; Boudreault et al., 2015) implies that $z_{0}$ over forest is larger than what has been typically assigned in wind resource assessment (i.e., $z_{0}>1$, not $z_{0} \lesssim 1$ ), despite such underestimates being used for decades in the wind industry (Troen and Petersen, 1989; Mortensen et al., 2001; Emeis, 2013; Landberg, 2016). We now see an explanation for this looking at Fig. 5: systematic underestimation leads to smaller errors in wind speeds predicted via the EWA method compared to a positive bias on $z_{0}$, particularly for typical application where both measurement and turbine sites are in highroughness areas (dash-dot line in Fig. 5) such as forest.

The roughness sensitivity-uncertainty analysis developed here also has application to - and implications on - the treatment of mesoscale model output for use in microscale wind flow models. In so-called meso-to-microscale downscaling or wind climate generalization (Hahmann et al., 2013; Badger et al., 2014), mesoscale wind output (or statistics of such) is treated in order to avoid "double-counting" of local surface-induced effects by the microscale model that have already been included in the mesoscale modeling. Additionally, the meso-micro downscaling procedure facilitates driving of the microscale flow simulation with mean winds that are appropriate as per the roughness input to both the microscale and mesoscale models, i.e., an effective geostrophic wind via the EWA method. Since any given planetary bound- ary layer (PBL) scheme in a mesoscale model can react differently for a given model resolution, it may be necessary to scale input roughnesses used in the generalization procedure (Kelly and Volker, 2016). For (homogeneous ideal) output wind profiles from a particular PBL scheme and resolution, the ratio of profile-implied $z_{0}$ to input $z_{0}$ can be used with the analytic sensitivity relations developed herein to systematically adjust the input roughness map and/or to scale the wind inputs to microscale models.

An additional application following from the roughness analysis herein - and consequently ongoing research - involves a limitation inherent in using a single characteristic (mean) roughness length. Due to the statistical nature of roughness and the significant width of measured roughness distributions (e.g., Fig. 1), an improvement would be to use $P\left(z_{0}\right)$ instead of mean $z_{0}$ in wind assessment and atmospheric flow modeling, following the suggestion of Kelly and Gryning (2010). This becomes yet more significant (and complicated) considering that the width of $P\left(z_{0}\right)$ tends to depend on direction and vary from site to site, and it also involves correlations with other variables (e.g., stability; Zilitinkevich et al., 2008). Given the limited applicability of the EWA method to time series (the GDL was not explicitly derived in a statistical mean sense), refined wind resource estimates - which are essentially statistical atmospheric fluid mechanics - using (joint) distributions of roughness and stability offer potential improvement over current mean methods and are a subject of continued study.

One final application follows from the analytical form introduced here to approximate common production power curves, in a general or universal way under the assumption of Weibull-distributed wind speeds. From this, the exponent in the power-law expression relating annual energy production and mean wind speed was derived, allowing us to relate uncertainty in roughness length to uncertainty in AEP. More flexible power-curve forms can also be made from logistic functions (e.g., generalizing those of Villanueva and Feijoo, 2016) as well. Regardless of the exact form, such analytical treatment also facilitates quick computation of power for a given set of Weibull parameters, which is applicable to large data sets such as the Global Wind Atlas (Badger et al., 2015). Lastly we re-iterate that issues in the definition of roughness length, and specific limits of its validity, are beyond the scope of this article. However, current ongoing work includes closer examination of the (turbulent) mechanisms involved in the observation of roughness length from wind measurements and heterogeneity; subsequent links to refined uncertainty characterization may follow such investigation.

\subsection{Summary of conclusions and implications}

- The EWA method (e.g., WAsP) exploits surface roughness information to improve resource predictions at one site based on measurements at another, but there is uncertainty $w_{z_{0}}$ in the roughness length. 
- Uncertainty in $z_{0}$ leads to uncertainty in predicting resources using the EWA method.

- Uncertainty in EWA-predicted mean wind depends upon $w_{z_{0}}$, and to a lesser extent also upon $\left\{z_{1}, z_{2}\right\}$.

- $w_{z_{0}}$ (half-width of $P\left(z_{0}\right)$ ) is of the same order as the mean, i.e., $w_{z_{0}} \sim\left\langle z_{0}\right\rangle$ for both user- and observationderived $z_{0}$.

- For modest $z_{0}$ uncertainties $\left(w_{z_{0}} \lesssim 2\left\langle z_{0}\right\rangle\right)$, the uncertainties $\{\Delta U, \Delta \mathrm{AEP}\} \propto\left[w_{z_{0}} /\left\langle z_{0}\right\rangle\right]^{6 / 7}$.

- In complex terrain and/or forest, ignoring the effect of form drag causes a positive bias in predictions.

- Underestimation of aggregate forest roughness leads to smaller error than overestimation.

- Analytical form for power curve $\operatorname{PC}\left(U / V_{\text {rat }}\right)$ gives $\operatorname{AEP}(\langle U\rangle)$ and thus uncertainty in $\mathrm{AEP}$, i.e., $\Delta \operatorname{AEP}(\Delta\langle U\rangle)$.

- EWA-GDL sensitivity expressions are applicable to treatment of WRF output for wind resources.

Data availability. The specific "'filtered" data used in this paper is going to be made available under www.neweuropeanwindatlas.eu in the near future. The data within Monte Carlo simulations is randomly generated via the equations/descriptions mentioned in this paper. 


\section{Appendix A: Geostrophic-roughness sensitivity relations: analytical forms and simplification}

Here we elucidate the relations and approximations that allow translation of the partial derivatives of hub-height wind speed with regard to roughness (i.e., Eq. 6) into sensitivity and uncertainty relations such as Eq. (8).

\section{A1 Sensitivity to measurement site roughness $z_{0,1}$}

First we approximate Eq. (6) by a modified power-law form that accounts for the strongest dependences $\left(z_{0,1}\right.$ and $\left.z_{\mathrm{obs}}\right)$, which we find to be

$$
\frac{\partial \ln U_{\text {pred }}}{\partial \ln z_{0,1}} \simeq 1.1 \frac{\left[\left(z_{\text {obs }} / z_{0,1}\right)\left(1+z_{\text {obs }} / 80 \mathrm{~m}\right)\right]^{-1 / 7}}{\ln \left(z_{\text {obs }} / z_{0,1}\right)} .
$$

This approximation is shown by the dotted lines in Fig. A1, which also shows that it closely matches Eq. (6).

Because the roughness uncertainty (in $\ln z_{0,1}$ space) may easily correspond to 3 or more times the reported (mean) $z_{0,1}$, one must integrate over $\ln z_{0,1}$ to find the relative uncertainty. Using Eq. (A1) and the substitution $x \equiv z_{\mathrm{obs}} / z_{0,1}$ we have

$$
\begin{aligned}
& \left.\Delta \ln U_{\text {pred }}\right|_{z_{0,1}} ^{a z_{0,1}}=\int_{z_{0,1}}^{a z_{0,1}} \frac{\partial \ln U_{2}}{\partial \ln z_{0,1}} \mathrm{~d} \ln z_{0,1}^{\prime} \\
& \simeq \int_{z_{0,1}}^{a z_{0,1}} \frac{1.1}{z_{\mathrm{obs}}} \frac{\left(z_{\mathrm{obs}} / z_{0,1}^{\prime}\right)^{6 / 7}}{\ln \left(z_{\mathrm{obs}} / z_{0,1}^{\prime}\right)} \frac{\mathrm{d} z_{0,1}^{\prime}}{\left(1+z_{\mathrm{obs}} / 80 \mathrm{~m}\right)^{1 / 7}} \\
& =1.1\left(1+\frac{z_{\mathrm{obs}}}{80 \mathrm{~m}}\right)^{-1 / 7} \int_{z_{\mathrm{obs}} /\left(a z_{0,1}\right)}^{z_{\mathrm{obs}} / z_{0,1}} \frac{x^{-8 / 7}}{\ln x} \mathrm{~d} x \\
& \left.\left.=\frac{1.1}{\left(1+z_{\mathrm{obs}} / 80 \mathrm{~m}\right)^{1 / 7}}{ }^{-1 / 7}\right]-\mathrm{li}\left[\left(\frac{z_{\mathrm{obs}}}{a z_{0,1}}\right)^{-1 / 7}\right]\right\} .
\end{aligned}
$$

Here $a$ is the fractional uncertainty in observationsite background roughness as in Eq. (9), i.e., $a \equiv\left(z_{0,1}+\right.$ $\left.\Delta z_{0,1}\right) / z_{0,1}$ so that $\Delta z_{0,1}=(a-1) z_{0,1}$. The analytical logarithmic integral function $\operatorname{li}(x) \equiv \int_{0}^{x}(\mathrm{~d} t / \ln t)$ can be evaluated using typical contemporary mathematical programming libraries, scientific analysis programs, or lookup-tables (Abramowitz and Stegun, 1972) ${ }^{8}$.

\section{A2 Sensitivity to prediction-site roughness $z_{0,2}$}

Just as above for the observation site background roughness, we can also express the uncertainty in predicted wind speed

\footnotetext{
${ }^{8}$ The error-scaling function can also be written in terms of the exponential integral function $\operatorname{Ei}(x) \equiv-\int_{-x}^{\infty}\left(e^{-t} \mathrm{~d} \ln t\right)$, i.e., $\operatorname{Ei}\left(\ln x^{-1 / 7}\right)$ evaluated at the same limits as in Eq. (A2).
}

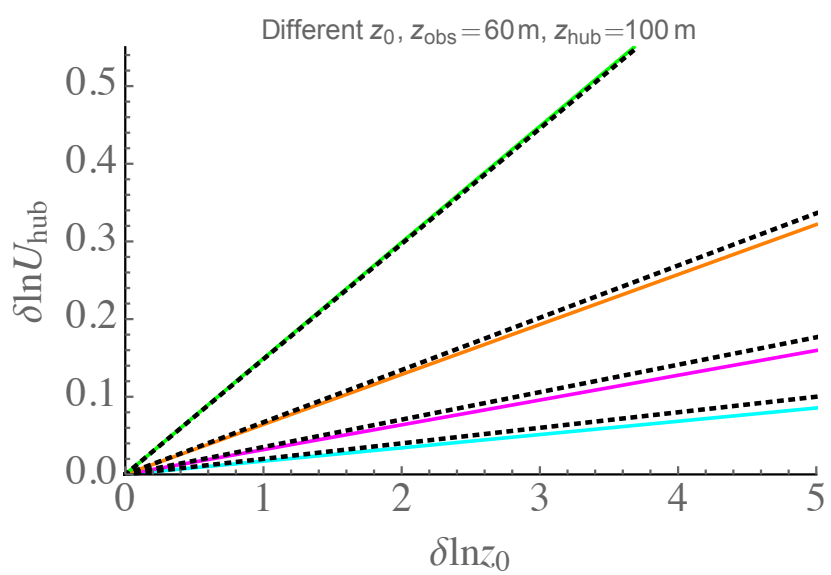

Figure A1. Equation (6) (solid) and its approximation, Eq. (A1) (dotted), for different (correctly observed) background roughnesses $z_{0, \text { obs }}$. Cyan: $z_{0,1}=0.001 \mathrm{~m}$; magenta: $z_{0,1}=0.01 \mathrm{~m}$; orange: $z_{0,1}=0.1 \mathrm{~m}$; green: $z_{0,1}=1 \mathrm{~m}$.

due to uncertainty in the roughness length for a prediction site. Following a similar procedure as above, using Eq. (7) and the substitution $y \equiv \ln z_{0,2}$ we obtain

$$
\begin{aligned}
& \left.\Delta\left(\ln U_{\text {pred }}\right)\right|_{z_{0,2}} ^{a z_{0,2}}=\int_{\ln \left(z_{0,2}\right)}^{\ln \left(a z_{0,2}\right)} \frac{\partial \ln U_{2}}{\partial \ln z_{0,2}} \mathrm{~d} \ln z_{0,2}^{\prime} \\
& \simeq \int_{y}^{y+\ln a} \frac{A+\ln \left(z_{\text {pred }} f / G\right)}{\left[y^{\prime}+\ln (f / G)+A\right]\left(\ln z_{\text {pred }}-y^{\prime}\right)} \mathrm{d} y^{\prime} \\
& =-\left.\ln \left[\frac{A+\ln (f / G)+y^{\prime}}{y^{\prime}-\ln z_{\text {pred }}}\right]\right|_{y} ^{y+\ln a} \\
& =\ln \left\{\frac{\left[1-\frac{\ln a}{\ln \left(z_{\text {pred }} / z_{0,2}\right)}\right]}{\left[1+\frac{\ln a}{A-\ln \left[G /\left(f z_{0,2}\right)\right.}\right]}\right\} .
\end{aligned}
$$

\section{A3 Sensitivity to heights of measurement and prediction}

Above it was written that predictions of wind speed (and thus AEP) were relatively insensitive to observation and measurement height, compared to the sensitivity to roughness. The minor dependence upon $z_{\text {obs }}$ in Eq. (A2) and upon $z_{\text {pred }}$ in Eq. (A3) is shown in Fig. A2 for the case of grassland at measurement and observation sites $\left(z_{0,1}=z_{0,2}=4 \mathrm{~cm}\right)$ as a function of roughness uncertainty in the form of $z_{0}$ bias.

As one can see from the figure, the EWA method, i.e., via the geostrophic drag law, predicted that $U$ has increased sensitivity to $\left\{z_{\mathrm{obs}}\right.$ and $\left.z_{\text {pred }}\right\}$ for large uncertainties in roughness length (biases in Fig. A2). However, even for a bias $a_{\text {bias }} \sim 3^{ \pm 1}(+200$ or $-67 \%)$, the resultant $\langle U\rangle$ uncertainty spans a range smaller than -1 to $2 \%$. For the case of independent uncertainties in $z_{0,1}$ and $z_{0,2}$, the half width of the associated $\Delta U$ distribution expands slightly, becoming roughly 


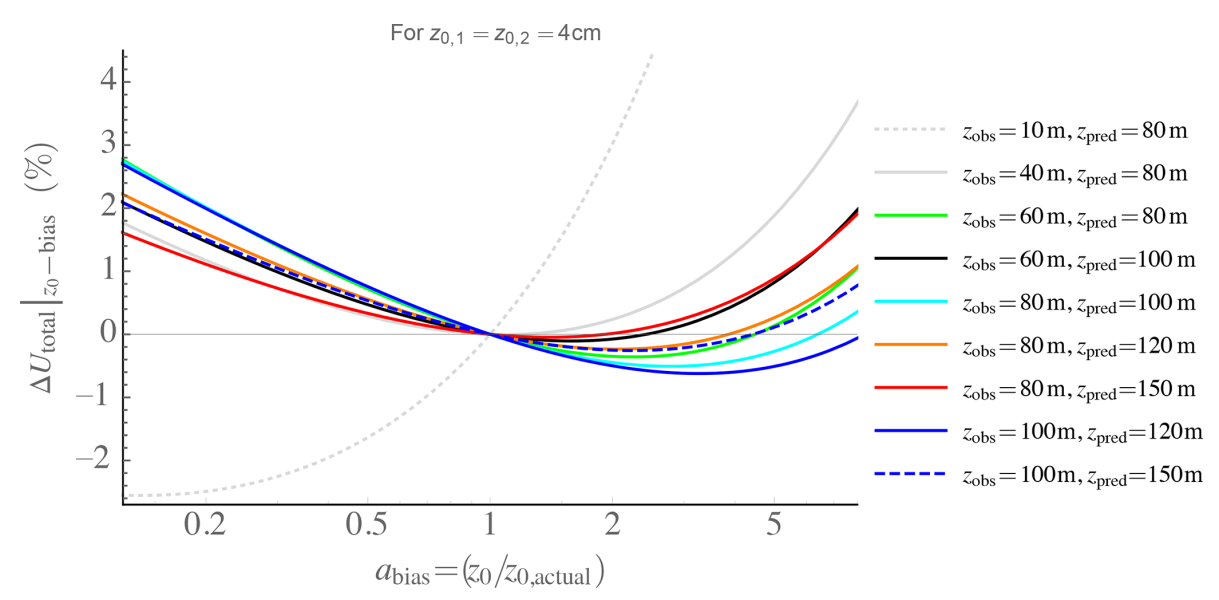

Figure A2. Total uncertainty vs. bias in background roughnesses $z_{0,1}$ and $z_{0,2}$ due to different combinations of measurement and prediction heights for the case of grassland $\left(z_{0}=4 \mathrm{~cm}\right)$ at both measurement and prediction sites.
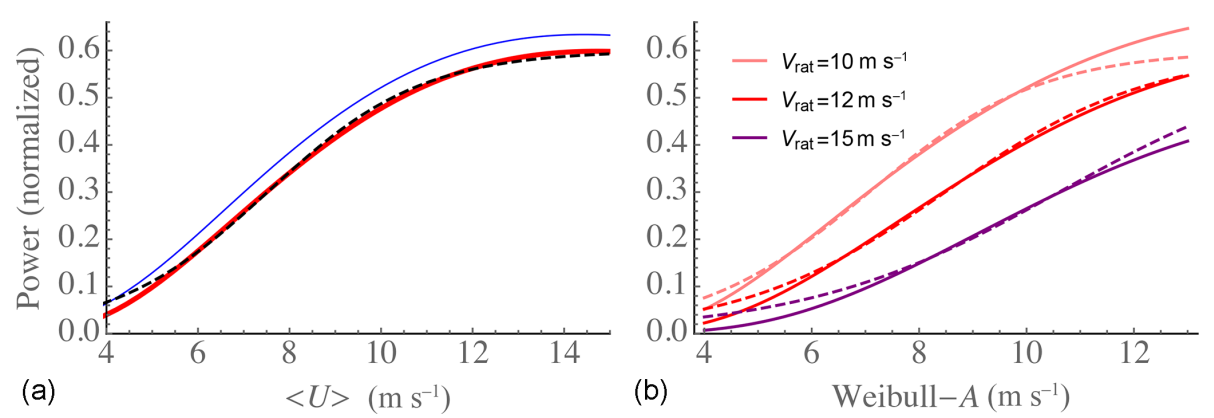

Figure A3. (a) Normalized power vs. mean wind speed for $k=2, V_{\text {rat }}=12 \mathrm{~m} \mathrm{~s}^{-1}$; blue is for ideal truncated (sharp) power curve, red is via numerically integrated universal power-curve form (Eq. B1), black dashed is approximation (Eq. B3) to universal form, and (green) dotted is for simple $U^{3}$ form. (b) Normalized power (convolution of Eq. B1 and Weibull distribution) as a function of Weibull- $A$ parameter for rated speeds of $10 \mathrm{~m} \mathrm{~s}^{-1}$ (pink), $12 \mathrm{~m} \mathrm{~s}^{-1}$ (red), and $15 \mathrm{~m} \mathrm{~s}^{-1}$ (purple); dashed lines indicate analytic approximation as in Eq. (B3).

$3 \%$ for an input roughness uncertainty $\left(\Delta z_{0} /\left\langle z_{0}\right\rangle\right.$ distribution half width) of 3 . These height-induced uncertainty values are small enough that one could use Fig. 8 for AEP uncertainty (where $z_{\text {obs }}=60 \mathrm{~m}$ and $z_{\text {pred }}=100 \mathrm{~m}$ ) and approximate the effect of varying $\left\{z_{\text {obs }}, z_{\text {pred }}\right\}$ from $\{60,100 \mathrm{~m}\}$ over simple terrain, by taking the difference between the curve for the desired $\left\{z_{\text {obs }}, z_{\text {pred }}\right\}$ and the $\{60,100 \mathrm{~m}\}$ curve in Fig. A2, and multiplying this by the effective $\operatorname{AEP}(\langle U\rangle)$ exponent $p$ (where the latter is detailed in the next appendix).

\section{Appendix B: Analytical power-curve forms for scalable calculation of AEP}

To propagate the uncertainty in mean wind speed into the annual energy production (AEP), it is necessary to have a model for AEP in terms of mean wind speed. Assuming a Weibull distribution for wind speeds, we are able to relate the Weibull parameters to AEP for a given power curve. In this appendix we produce a universal power-curve formulation, which allows us to derive an expression for conversion of Weibull- $A$ parameter (or mean wind speed) into AEP for any given tur- bine rated speed $V_{\text {rat }}$. The forms we provide here apply for wind speed distributions with a Weibull-shape $(k)$ parameter of roughly 2; such Rayleigh-distributed mean winds tend to be the most commonly found (i.e., $k \approx 2$ tends to be most likely; see Troen and Petersen, 1989; Kelly et al., 2014b).

A canonical form for power curves including the smooth transition from ideal to maximum power for mean winds approaching rated speed $V_{\text {rat }}$ is

$$
\begin{aligned}
& \operatorname{PC}\left(U / V_{\text {rat }}\right)= \\
& P_{0} \times\left\{\frac{1}{2}+\frac{1}{2} \tanh \left[\pi\left(U / V_{\text {rat }}-n^{-1 / 2}\right)\right]\right\}^{n}, \quad n=3 .
\end{aligned}
$$

We choose the order $n$ to be 3, matching the ideal $U^{3}$ behavior in the regime for wind speeds above cut-in and below rated wind speed. Convolving Eq. (B1) with the Weibull probability density for wind speed

$$
f(U)=\frac{k}{U}\left(\frac{U}{A}\right)^{k} \exp \left[-\left(\frac{U}{A}\right)^{k}\right]
$$



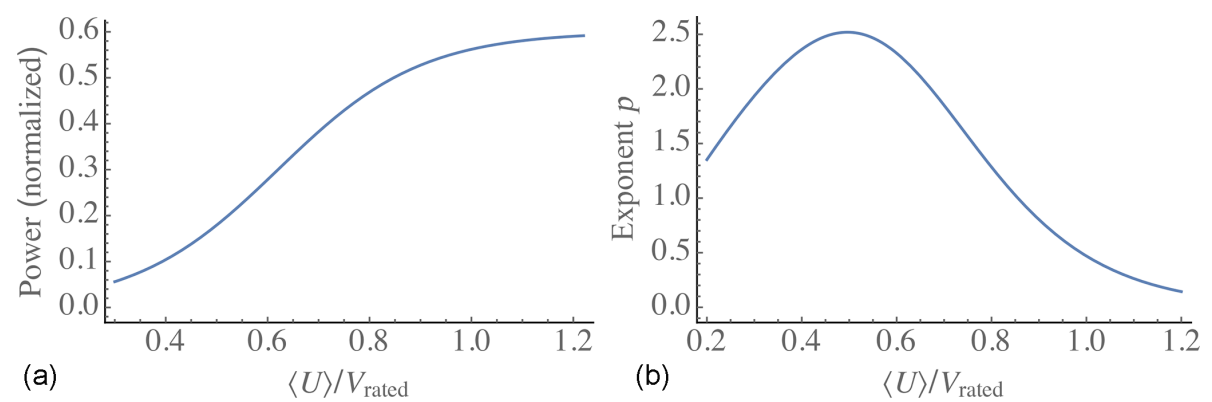

Figure A4. (a) Normalized AEP vs. mean wind relative to rated wind speed. (b) AEP effective power-law exponent vs. mean wind over rated speed, obtained via integrable power-law form (Eq. B1) and subsequent dimensionless AEP (Eq. B3) for Weibull-distributed wind with $k=2$.

gives the normalized AEP, but this is not quite amenable to (simple) analytical relation. Thus, in order to find a useful (closed) expression for the AEP, we make an approximation to the convolution $\int f(U) \mathrm{PC}(U) \mathrm{d} U$ via Eqs. (B1) and (B2):

$$
\frac{\mathrm{AEP}}{\mathrm{AEP}_{0}} \simeq 0.3\left\{1+\tanh \left[\pi\left(\frac{A}{V_{\text {rat }}}-\frac{1}{\sqrt{2}}\right)\right]\right\} .
$$

The closed-form approximation (Eq. B3) for normalized AEP is shown in Fig. A3, along with the numerically integrated product of Eqs. (B1) and (B2), which it approximates, for the case of Rayleigh-distributed wind speeds $(k=2)$. The left-hand plot (Fig. A3a) gives $\mathrm{AEP} / \mathrm{AEP}_{0}$ as a function of mean wind speed $\langle U\rangle^{9}$ for a single value of $V_{\text {rat }}$ and also displays the results corresponding to use of either a simple $P \propto U^{3}$ power curve, or an ideally limited power curve that has $\mathrm{PC}(U) / \mathrm{PC}_{\text {rat }}=\left\{\left(U / V_{\text {rat }}\right)^{3}, 1\right\}$ for $\left\{U<V_{\text {rat }}, U \geq V_{\text {rat }}\right\}$. Figure $\mathrm{A} 3 \mathrm{~b}$ again shows the numerically integrated and approximated nominal power, but as a function of Weibull- $A$ and for different $V_{\text {rat }}$.

One can see from Fig. A3 that the approximation (Eq. B3) works well for mean wind speeds and rated speeds typical of multi-megawatt turbines (and associated hub heights), i.e., $\langle U\rangle \sim 6-14$ and $V_{\text {rat }} \sim 12-15 \mathrm{~m} \mathrm{~s}^{-1}$.

Most succinctly, given a Weibull- $A$ value (or mean wind speed) and turbine-rated speed $V_{\text {rat }}$, the AEP can be simply estimated by Eq. (B3) as a function of $A / V_{\text {rat }}$; this is shown in Fig. A4a.
The effective wind-power exponent $p$ defined by AEP $=$ $U^{p}$ can now be found analytically from the corresponding analytical form (Eq. B3) for normalized AEP:

$$
\begin{aligned}
p & =\frac{\ln \mathrm{AEP}}{\ln \langle U\rangle}=\frac{\partial \ln \mathrm{AEP}}{\partial \ln \langle U\rangle} \\
& =\frac{\pi\left(A / V_{\mathrm{rat}}\right) \operatorname{sech}^{2}\left[\pi\left(A / V_{\mathrm{rat}}-2^{-1 / 2}\right)\right]}{1+\tanh \left[\pi\left(A / V_{\mathrm{rat}}-2^{-1 / 2}\right)\right]} .
\end{aligned}
$$

The power-law exponent derived in Eq. (B4) is displayed in Fig. A4b for the case of Weibull-shape parameter $k=2$. Evident from the figure is the optimal choice of sites with mean winds at hub height that are $\sim 60-80 \%$ of rated speed, as well as the diminishing returns that can result from using turbines with rated speeds not much higher than the mean wind speed.

For a given value of $\langle U\rangle / V_{\text {rat }}$, via $\mathrm{AEP} \propto U^{p}$ and Eq. (B4), we are able to translate uncertainty in mean wind speed estimates (due to background roughness, for example) into AEP uncertainty.

\footnotetext{
${ }^{9}$ For Rayleigh-distributed wind speeds (Weibull, with $k=2$ ), the mean wind is simply $\langle U\rangle=A \Gamma(1+1 / k) \simeq 0.89 A$.
} 
Competing interests. The authors declare that they have no conflict of interest.

Acknowledgements. The authors would like to thank the reviewers for their time and effort towards constructive criticism of the present article. Mark Kelly is also grateful to Andrey Sogachev for discussion and for pointing toward the Bosveld (1997) and Tian et al. (2011) works. Mark Kelly further thanks Neil Davis for updates on logistic-function use in wind energy and for testing the analytical power-curve form on some big data sets.

Edited by: H. Hangan

Reviewed by: two anonymous referees

\section{References}

Abramowitz, M. and Stegun, I. A.: Handbook of Mathematical Functions with Formulas, Graphs, and Mathematical Tables, 9th Edn., Dover, New York, 1972.

Badger, J., Frank, H., Hahmann, A. N., and Giebel, G.: WindClimate Estimation Based on Mesoscale and Microscale Modeling: Statistical-Dynamical Downscaling for Wind Energy Applications, J. Appl. Meteorol. Clim., 53, 1901-1919, doi:10.1175/JAMC-D-13-0147.1, 2014.

Badger, J., Davis, N., Hahmann, A. N., Olsen, B. T., Larsén, X. G., Kelly, M., Volker, P., Badger, M., Ahsbahs, T. T., Mortensen, N. G., Ejsing Jørgensen, H., Lundtang Petersen, E., Lange, J., and Fichaux, N.: The new worldwide microscale wind resource assessment data on IRENA's Global Atlas, The EUDP Global Wind Atlas, in: EWEA Technology Workshop 2015, European Wind Energy Association (EWEA), Helsinki, Finland, 2-3 June, 2015.

Beljaars, A. C. M., Brown, A. R., and Wood, N.: A new parametrization of turbulent orographic form drag, Q. J. Roy. Meteor. Soc., 130, 1327-1347, 2004.

Bosveld, F. C.: Derivation of fluxes from profiles over a moderately homogeneous forest, Bound.-Lay. Meteorol., 84, 289-327, 1997.

Bou-Zeid, E., Meneveau, C., and Parlange, M.: Large-eddy simulation of neutral atmospheric boundary layer flow over heterogeneous surfaces: blending height and effective surface roughness, Water Resour. Res., 40, W02505, doi:10.1029/2003WR002475, 2004.

Boudreault, L.-E., Bechmann, A., Tarvainen, L., Klemedtsson, L., Shendryk, I., and Dellwik, E.: A LiDAR method of canopy structure retrieval for wind modeling of heterogeneous forests, Agr. Forest Meteorol., 201, 86-97, doi:10.1016/j.agrformet.2014.10.014, 2015.

Calaf, M., Higgins, C., and Parlange, M.: Large Wind Farms and the Scalar Flux over an Heterogeneously Rough Land Surface, Bound.-Lay. Meteorol., 153, 471-495, 2014.

Clerc, A., Anderson, M., and Stuart, P.: A systematic method for quantifying wind flow modelling uncertainty in wind resource assessment, J. Wind Eng. Ind. Aerod., 111, 85-94, 2012.

Danish Geodata Agency: Danmarks højdemodel (Danish terrain elevation model), available at: http://www.gst.dk/media/gst/ 2641297/5FAQ_v1_2.pdf, 2015 (in Danish).
Ellison, T. H.: Atmospheric Turbulence, in: Surveys in mechanics: A collection of surveys of the present position of research in some branches of mechanics, written in commemoration of the 70th birthday of G. I. Taylor, edited by: Batchelor, G. and Davies, R., University press, 475 pp., 1956.

Emeis, S.: Wind energy meteorology - Atmospheric Physics for wind power generation, Springer, Dordrecht, 2013.

Floors, R. R., Kelly, M., Troen, I., Pena Diaz, A., and Gryning, S.E.: Wind profile modelling using WAsP and "tall" wind measurements, in: Abstracts of the 15th EMS Annual Meeting, European Meteorological Society, 2015.

Garratt, J. R.: Transfer characteristics for a heterogeneous surface of large aerodynamic roughness, Q. J. Roy. Meteor. Soc., 104, 491-502, 1978.

Hahmann, A. N., Casso, P., Campmany, E., Vincent, C. L., Kelly, M. C., Badger, J., Moreno, P., and Ejsing Jørgensen, H.: Towards a generalization procedure for WRF mesoscale wind climatologies, European Wind Energy Conference 2013, European Wind Energy Association (EWEA), Vienna, Austria, 4-7 February, 2013

Hahmann, A. N., Lennard, C., Badger, J., Vincent, C. L., Kelly, M., Volker, P. J., Argent, B., and Refslund, J.: Mesoscale modeling for the Wind Atlas of South Africa (WASA) project, Tech. Rep. DTU Wind Energy E-0050(EN), Wind Energy Dept., Ris $\varnothing$ Lab/Campus, Danish Tech. Univ. (DTU), Roskilde, Denmark, 2015.

Hasager, C. B. and Jensen, N. O.: Surface-flux aggregation in heterogeneous terrain, Q. J. Roy. Meteor. Soc., 125, 2075-2102, 1999.

Högstrom, U.: Review of some basic characteristics of the atmospheric surface layer, Bound.-Lay. Meteorol., 78, 215-246, 1996.

Jensen, N. O., Petersen, E. L., and Troen, I.: Extrapolation of Mean Wind statistics with Special Regard to Wind Energy Applications, World climate programme report, WMO, Geneva, Switzerland, report no. WCP-86, 1984.

Kelly, M.: Uncertainty in vertical extrapolation of wind statistics: shear-exponent and WAsP/EWA methods, Tech. Rep. DTU Wind Energy E-0121(EN), Wind Energy Dept., Ris $\emptyset$ Lab/Campus, Danish Tech. Univ. (DTU), Roskilde, Denmark, 2016.

Kelly, M. and Gryning, S.-E.: Long-Term Mean Wind Profiles Based on Similarity Theory, Bound.-Lay. Meteorol., 136, 377390, 2010.

Kelly, M. and Jørgensen, H. E.: Uncertainties in AEP due to the uncertainty in the roughness estimation- A simplified analytic approach, in: Vindkraft-net: Uncertainty (2014, COWI, Copenhagen), Danish Research Consortium for Wind Energy, available at: http://windpower.org/da/aktiviteter/afholdte_aktiviteter/ vindkraftnet_events_2009-2015.html\#none, 2014.

Kelly, M. and Troen, I.: Probabilistic stability and "tall" wind profiles: theory and method for use in wind resource assessment, Wind Energy, 19, 227-241, 2016.

Kelly, M. and Volker, P.: WRF idealized-roughness response: PBL scheme and resolution dependence, Abstracts of the 16th EMS Annual Meeting, European Meteorological Society, 2016.

Kelly, M., Larsen, G., Dimitrov, N. K., and Natarajan, A.: Probabilistic Meteorological Characterization for Turbine Loads, J. Phys. Conf. Ser., 524, 012076, doi:10.1088/17426596/524/1/012076, 2014a. 
Kelly, M., Troen, I., and Jørgensen, H. E.: Weibull- $k$ revisited: “tall” profiles and height variation of wind statistics, Bound.-Lay. Meteorol., 152, 107-124, 2014b.

Kiureghian, A. D. and Ditlevsen, O.: Aleatory or epistemic? Does it matter?, Struct. Saf., 31, 105-112, 2009.

Krishna, K.: The planetary-boundary-layer model of Ellison (1956) - A retrospect, Bound.-Lay. Meteorol., 19, 293-301, 1980.

Landberg, L.: Meteorology for wind energy: an introduction, John Wiley \& Sons Ltd., UK, 2016.

Lettau, H.: Note on aerodynamic roughness-parameter estimation on the basis of roughness-element description, J. Appl. Meteor., 8, 828-832, 1969.

Liu, S. and Liang, X.-Z.: Observed Diurnal Cycle Climatology of Planetary Boundary Layer Height, J. Climate, 23, 5790-5809, 2010.

Marticorena, B., Kardous, M., Bergametti, G., Callot, Y., Chazette, P., Khatteli, H., Le Hégarat-Mascle, S., Maillé, M., Rajot, J.L., Vidal-Madjar, D., and Zribi, M.: Surface and aerodynamic roughness in arid and semiarid areas and their relation to radar backscatter coefficient, J. Geophys. Res.-Earth, 111, F03017, doi:10.1029/2006JF000462, 2006.

Monin, A. S. and Yaglom, A. M.: Statistical Fluid Mechanics, Vol. 1, The MIT Press, 1971.

Mortensen, N., Heathfield, D. N., Rathmann, O., and Nielsen, M.: Wind Atlas Analysis and Application Program: WAsP 11 Help Facility, 2014.

Mortensen, N. G., Landberg, L., Rathmann, O., Frank, H. P., Troen, I., and Petersen, E. L.: Wind atlas analysis and application program (WAsP), Report, Risø National Laboratory, Roskilde, Denmark, 2001.

Mortensen, N. G., Bowen, A. J., and Antoniou, I.: IMPROVING WAsP PREDICTIONS IN (TOO) COMPLEX TERRAIN, in: European Wind Energy Conference 2013, European Wind Energy Association (EWEA), Athens, Greece, 2006.

Nikuradse, J.: Strömungsgesetze in rauhen Rohren, Forschungsheft, 4, 1933.

Pedersen, J. G., Gryning, S.-E., and Kelly, M.: On the Structure and Adjustment of Inversion-Capped Neutral Atmospheric Boundary-Layer Flows: Large-Eddy Simulation Study, Bound.Lay. Meteorol., 153, 43-62, doi:10.1007/s10546-014-9937z,2014.

Sempreviva, A. M., Larsen, S. E., Mortensen, N. G., and Troen, I.: Response of neutral boundary layers to changes in roughness, Bound.-Lay. Meteorol., 50, 205-225, 1990.

Sogachev, A. and Kelly, M.: On Displacement Height, from Classical to Practical Formulation: Stress, Turbulent Transport and Vorticity Considerations, Bound.-Lay. Meteorol., 158, 361-381, doi:10.1007/s10546-015-0093-x, 2016.
Sørensen, N. N., Bechmann, A., Rethore, P.-E., Cavar, D., Kelly, M., and Troen, I.: How fine is fine enough when doing CFD terrain simulations, European Wind Energy Association (EWEA), 2012.

Stull, R. B.: Introduction to boundary layer meteorology, Kluwer, 1988.

Tian, X., Li, Z., van der Tol, C., Su, Z., Li, X., He, Q., Bao, Y., Chen, E., and Li, L.: Estimating zero-plane displacement height and aerodynamic roughness length using synthesis of LiDAR and SPOT-5 data, Remote Sens. Environ., 115, 2330-2341, 2011.

Torbick, N., Lusch, D., Qi, J., Moore, N., Olson, J., and Ge, J.: Developing land use/land cover parameterization for climate-land modelling in East Africa, Int. J. Remote Sens., 27, 4227-4244, doi:10.1080/01431160600702426, 2006.

Tripp, W.: Friction losses in artificially roughened rectangular channel, J. Aeronaut. Sci., 4, 10-11, 1936.

Troen, I. and Petersen, E. L.: European Wind Atlas, Risø National Laboratory, Roskilde, Denmark, 1989.

Troen, I., Bechmann, A., Kelly, M., Sørensen, N. N., Réthoré, P.-E., Cavar, D., and Ejsing Jørgensen, H.: Complex terrain wind resource estimation with the wind-atlas method: Prediction errors using linearized and nonlinear CFD micro-scale models, in: Proceedings of the 2014 EWEA conference, European Wind Energy Association (EWEA), Barcelona, 2014.

Varian, H.: Bootstrap Tutorial, Mathematica J., 9, 768-775, 2005.

Villanueva, D. and Feijoo, A. E.: Reformulation of parameters of the logistic function applied to power curves of wind turbines, Electr. Pow. Syst. Res., 137, 51-58, doi:10.1016/j.epsr.2016.03.045, 2016.

Wagner, R., Courtney, M., Gottschall, J., and Lindelow-Marsden, P.: Accounting for the speed shear in wind turbine power performance measurement, Wind Energy, 14, 993-1004, doi:10.1002/we.509, 2011.

Walmsley, J. L.: Proposal for new PBL resistance laws for neutrallystratified flow, Bound.-Lay. Meteorol., 60, 271-306, 1992.

$\mathrm{Wu}, \mathrm{C}$. .: Jackknife, bootstrap and other resampling methods in regression analysis (with discussions), Ann. Stat., 14, 1261-1350, 1986.

Wyngaard, J. C.: Turbulence in the Atmosphere, Cambridge University Press, 2010.

Zhang, K., Whitman, D., Leatherman, S., and Robertson, W.: Quantification of beach changes caused by Hurricane Floyd along Florida's Atlantic coast using airborne laser surveys, J. Coastal Res., 21, 123-134, 2005.

Zilitinkevich, S. S., Mammarella, I., Baklanov, A. A., and Joffre, S. M.: The effect of stratification on the aerodynamic roughness length and displacement height, Bound.-Lay. Meteorol., 129, 179-190, doi:10.1007/s10546-008-9307-9, 2008. 